\title{
Comparison of Whiskbroom and Pushbroom darkfield elastic light scattering spectroscopic imaging for head and neck cancer identification in a mouse model
}

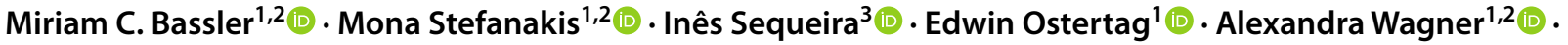 \\ Jörg W. Bartsch ${ }^{4}$ - Marion Roeßler ${ }^{5} \cdot$ Robert Mandic $^{6}$ (D) Eike F. Reddmann ${ }^{1}$ (D) . Anita Lorenz ${ }^{1}$ (D) \\ Karsten Rebner ${ }^{1} \mathbb{D} \cdot$ Marc Brecht $^{1,2} \mathbb{D}$
}

Received: 8 June 2021 / Revised: 30 August 2021 / Accepted: 8 October 2021 / Published online: 19 November 2021

(c) The Author(s) 2021, corrected publication 2022

\begin{abstract}
The early detection of head and neck cancer is a prolonged challenging task. It requires a precise and accurate identification of tissue alterations as well as a distinct discrimination of cancerous from healthy tissue areas. A novel approach for this purpose uses microspectroscopic techniques with special focus on hyperspectral imaging (HSI) methods. Our proof-ofprinciple study presents the implementation and application of darkfield elastic light scattering spectroscopy (DF ELSS) as a non-destructive, high-resolution, and fast imaging modality to distinguish lingual healthy from altered tissue regions in a mouse model. The main aspect of our study deals with the comparison of two varying HSI detection principles, which are a point-by-point and line scanning imaging, and whether one might be more appropriate in differentiating several tissue types. Statistical models are formed by deploying a principal component analysis (PCA) with the Bayesian discriminant analysis (DA) on the elastic light scattering (ELS) spectra. Overall accuracy, sensitivity, and precision values of $98 \%$ are achieved for both models whereas the overall specificity results in $99 \%$. An additional classification of model-unknown ELS spectra is performed. The predictions are verified with histopathological evaluations of identical HE-stained tissue areas to prove the model's capability of tissue distinction. In the context of our proof-of-principle study, we assess the Pushbroom PCA-DA model to be more suitable for tissue type differentiations and thus tissue classification. In addition to the HE-examination in head and neck cancer diagnosis, the usage of HSI-based statistical models might be conceivable in a daily clinical routine.
\end{abstract}

Keywords Mie elastic light scattering spectroscopy $\cdot$ Chemometrics/statistics $\cdot$ Clinical/biomedical analysis $\cdot$ Head and neck cancer $\cdot$ Mouse tumor model $\cdot$ Microspectroscopy

Marc Brecht

marc.brecht@ reutlingen-university.de

1 Process Analysis and Technology (PA\&T), Reutlingen University, Alteburgstr. 150, 72762 Reutlingen, Germany

2 Institute of Physical and Theoretical Chemistry, University of Tübingen, Auf der Morgenstelle 18, 72076 Tübingen, Germany

3 Institute of Dentistry, Barts and the London School of Medicine and Dentistry, Queen Mary University of London, London, UK

4 Department of Neurosurgery, Philipps University Marburg, Baldingerstraße, 35033 Marburg, Germany

5 Department of Pathology, Philipps University Marburg, Baldingerstraße, 35033 Marburg, Germany

6 Department of Otorhinolaryngology, Philipps University Marburg, Baldingerstraße, 35033 Marburg, Germany

\section{Introduction}

Microspectroscopic imaging is a powerful tool to investigate biological materials of any kind and helps to reveal their structure, functionality, and purpose within the organism. It correlates the spectroscopic data with the microscopic image and thus allows for a spatial assignment of spectral information. Important biological applications for microspectroscopic imaging, such as Raman, fluorescence, or infrared (IR) imaging, are the analysis of cell systems and tissues [1, 2], particularly in cancer research and diagnosis [3].

Despite intense research and therapy development, cancer still belongs to one of the most threatening diseases humankind is suffering. In 2018, approximately $18.1 \mathrm{mil}-$ lion new cancer cases were estimated worldwide with a higher incidence in lung cancer, female breast cancer, and 
colorectal cancer [4], closely followed by head and neck squamous cell carcinomas (HNSCC), as the sixth most common cancer type [5]. HNSCC encompass a variety of tumors originating in the lip, oral cavity, hypopharynx, oropharynx, nasopharynx, or larynx. Oral squamous cell carcinomas (OSCC), a subset of HNSCC, account for 355,000 new cases annually worldwide [6] with a 5-year survival rate of $50 \%$ [7]. In recent studies, significant inter-tumoral heterogeneity was observed by histopathology, reflecting the tumor site of origin, proliferation, the grade of differentiation, depth of invasion, and degree of inflammation [8]. Oral cancer screening is inevitable for early detection and early treatment of tumors, which could considerably improve survival rates.

Early detection of HNSCC requires sensitive identification and localization methods, able to measure small cell and tissue changes. So far, hematoxylin-eosin staining (HE-staining) represents the gold standard in histopathology to recognize head and neck (HN) lesions in several stages during carcinogenesis. Additional early detection tools for HNSCC comprise spectroscopic technologies like narrowband imaging (NBI) [9], Raman [10], and fluorescence spectroscopy [11]. One spectroscopic technique demonstrated exceptional suitability to early detect preneoplastic variations during colorectal cancer genesis, which is elastic light scattering spectroscopy (ELSS) [12]. Already 2 weeks prior to the first evidence of malignant tissue alteration, marked changes were detectable by measuring nano- and microscale architectures of the colonic tissue with elastic light scattering (ELS) [12]. On the cellular level, ELSS elucidates morphological features such as size distribution of cells and nuclei or the degree of nuclei pleomorphism and hyperchromasia [13]. For the morphological tissue characterization and differentiation, great achievements with ELSS could not only be generated in colorectal cancer [14], but also in breast cancer [15], skin cancer [16], brain tumors [17], and most importantly in HNSCC [18]. Recently, our group demonstrated to distinguish formalin-fixed brain tumor tissues with a varying degree of malignancy by optical spectroscopy, including ELSS [19].

The implementation of ELSS as an imaging method allows for the combination of spatial (x,y-direction) with spectral information ( $\lambda$-direction). This results in a $3 \mathrm{D}$ data matrix called a hypercube $[20,21]$. The hypercube can be evaluated in two ways: image planes can either be extracted at certain wavelength bands or the whole spectrum of one $\mathrm{x}, \mathrm{y}$-coordinate or pixel is used within the image plane [22]. Various studies of cancer diagnosis with HSI were accomplished [23-25]. HSI has yet been investigated in terms of its overall suitability in cancer detection and diagnosis using different cancer and sample types, spectral ranges, light sources, acquisition modes, or evaluation algorithms [21].
Most groups so far concentrated on the successful classification of different tissue or cell types to make it useable in cancer surgery. Our study, however, aims to investigate, for the first time, ELSS with two varying HSI detection principles, which are Whiskbroom and Pushbroom imaging, in order to compare their ability to detect ELS of predefined tissue samples. We want to verify whether the time-consuming pointby-point measurement of the Whiskbroom imaging is more suitable for ELS detection than the line-scanning system of a Pushbroom imager since both imaging methods exhibit different lateral and spectral resolution capabilities [21]. With respect to data acquisition, we modified our instrumental setups by installing a darkfield (DF) illumination pathway according to Ostertag et al. [26, 27]. Our adaption consequently enables darkfield elastic light scattering (DF ELS) as a non-contact microspectroscopic imaging modality. For a better understanding and comparability of both scanning techniques, advantages and disadvantages are summarized in Table 1.

As a proof-of-concept study, DF ELS Whiskbroom and Pushbroom imaging are applied on a HNSCC mouse model analyzing longitudinal-cut tissue sections of mouse tongues. The obtained spectral images are analyzed using multivariate data analysis (MVA). By combining a principal component analysis (PCA) and Bayesian discriminant analysis (DA), we generate two statistical models of the tongue tissue data based on both HSI detection techniques and compare if one of the models achieves better results in discriminating different tongue tissue types. Based on this discrimination, we derive whether noticeable differences between Whiskbroom and Pushbroom imaging appear. Finally, the performance of both statistical models is determined and verified regarding their ability to correctly classify model-unknown ELS spectra of varying tissue types. An additional comparison of the statistical model performance with classical HE histopathology is accomplished. If proven reliable, the statistical models could help to provide diagnostic information for physicians during surgery.

\section{Materials and methods}

\section{Mouse model for OSCC and carcinogenesis}

For this proof-of-concept study, we used an autologous mouse model of OSCC. A total amount of four different mice was investigated. Mice were subdivided into two control mice and two mice developing tumors. Control mice (mice A and B) exhibit an intact tissue structure. Therefore, mice A and B mainly provide healthy epithelium specimens as well as glandular and muscle tissue. Mice with dysplastic alterations (mice $\mathrm{C}$ and $\mathrm{D}$ ) display several stages of tissue modification like hyperplastic and dysplastic areas or 
Table 1 Advantages and disadvantages of Whiskbroom (column 2) and Pushbroom (column 3) imaging listed by several criteria (column 1).

The following overview should emphasize the strength of each individual imaging method and point out customized features of the setups used in this study. Column 4 lists the references to each feature

\begin{tabular}{|c|c|c|c|}
\hline Criteria & Whiskbroom imaging & Pushbroom imaging & References \\
\hline Spectral resolution & High & High & {$[20,21]$, customized } \\
\hline Spatial resolution & High & High & [28] \\
\hline Scanning speed & Time-consuming & Fast & [21] \\
\hline Field of measurement & $\begin{array}{l}\text { Restricted } \\
(150 \mu \mathrm{m} \times 150 \mu \mathrm{m}) \\
\text { (by piezo table) }\end{array}$ & $\begin{array}{l}\text { Large } \\
(340 \mu \mathrm{m} \times \text { several mm }) \\
(\mathrm{x} \text {-direction } \times \text {-direc- } \\
\text { tion })\end{array}$ & Customized \\
\hline Spectrometer entrance & Pinhole-based & Slit-based & Customized \\
\hline Spatial aliasing & No & Yes & [21], customized \\
\hline Acquisition mode & Off-line & On-line/in-line & {$[20]$} \\
\hline Splitting of light & $\begin{array}{l}\text { uniformly high efficiency due } \\
\text { to dispersive elements }\end{array}$ & $\begin{array}{l}\text { Uniformly high effi- } \\
\text { ciency due to disper- } \\
\text { sive elements }\end{array}$ & {$[28]$} \\
\hline Costs & $\begin{array}{l}\text { High } \\
\geq 100,000 €\end{array}$ & $\begin{array}{l}\text { Low } \\
\leq 100,000 €\end{array}$ & [28], customized \\
\hline Hardware & Complex & Complex & [28] \\
\hline
\end{tabular}

invasive squamous cell carcinomas (SCC). Modified tissue, mainly composed of invasive SCC, was derived from mouse C. Altered tissue specimens of mouse D function as prediction areas for the statistical model validation. Mice were maintained on the $\mathrm{C} 57 \mathrm{Bl} / 6 \mathrm{~N}$ genetic background.

Tumors are induced by chronic oral administration of the water-soluble carcinogen 4-nitroquinoline-1-oxide (4NQO) $[8,29,30]$ that mimics the alterations caused by tobacco mutagens. 4NQO forms DNA adducts, causing substitution of adenosine for guanosine, and induces intracellular oxidative stress resulting in mutations and DNA strand breaks [31]. Additionally, 4NQO is known to induce point mutations in HRas with subsequent loss of heterozygosity [32], upregulation of EGFR [33], p53 mutations [8], and reduced expression of the cell cycle inhibitor p16 [33]. These effects are similar to the genetic alterations induced by tobacco carcinogens [8, 33]. As in human OSCC, invasive tumors are preceded by epithelial hyperplasia and dysplasia [8].

Mice from both genders were maintained on the C57BL/6 N genetic background and were housed under a 12-h light/12-h dark cycle, at temperatures of $20-24{ }^{\circ} \mathrm{C}$ with $45-65 \%$ humidity. Starting weights of the mice ranged between $25 \mathrm{~g}$ and $32 \mathrm{~g}$. 4NQO (Sigma, diluted to $100 \mu \mathrm{g}$ / $\mathrm{mL}$ in water) was administered in the drinking water and changed once a week for 16 weeks (Fig. 1a). After that period, $\mathrm{C} 57 \mathrm{Bl} / 6 \mathrm{~N}$ mice were given drinking water without 4NQO. Mice were maintained with regular mouse chow and water $( \pm 4 \mathrm{NQO})$ ad libitum. Once a week, 4NQO-treated mice were sedated with inhaled isoflurane and the oral cavities were screened for lesions (hyperplasias, dysplasias, and SCCs) [8, 30]. All animal procedures were subject to institutional ethical review and approved by the UK Home Office (in accordance with UK law, Animals Scientific Procedures
Act 1986) at King's College London prior to commencement (Project license number 70/8474). We adhere to ARRIVE guidelines as set out by the NC3Rs.

Tongue tissues were harvested and embedded in OCT (optimal cutting temperature compound, VWR). Sequential cross sections were cut using a cryostat (CryoStar NX50, ThermoFisher) at $10 \mu \mathrm{m}$ thickness and post-fixed in $3.7 \%$ paraformaldehyde/PBS $\mathrm{pH} 7.4$ for $15 \mathrm{~min}$, washed twice in PBS and air-dried before staining. All murine tongues were cut equally in a longitudinal cutting direction. For DF ELS imaging, tissue sections were transferred to gold-coated (BioGold $^{\mathrm{TM}} 100 \mathrm{~nm}$ coat thickness, Thermo Scientific ${ }^{\mathrm{TM}}$ ) microarray slides. Comparable tissue cross sections were additionally placed on glass objective slides and HE-stained by conventional methods. By HE-staining, lesions were identified and classified macroscopically and microscopically. We found lesions on the dorsal and ventral tongue (Fig. 1b, c) and some animals presented more than one lesion. HEimages were acquired using a Hamamatsu slide scanner and analyzed using NanoZoomer software (Hamamatsu).

Tumor grading was assessed according to the presence of the following criteria: tumor cell crowding, degree of keratinization, exophytic or invasive growth, scattered mitotic figures, and nuclear atypia [34]. All histological assessments were performed by a pathologist blinded to the study groups and $4 \mathrm{NQO}$ treatment conditions.

Owing to the great heterogeneity of tumors and modified tissues [35], we refrain from distinguishing several stages of carcinogenesis, but define all types of tumor alterations, including hyperplasia, dysplasia, and invasive SCC, as altered tissue (AT) (Fig. 1c). The healthy counterpart consists of the epithelium (EP) and a mixture of glandular and muscle tissue (GM) (Fig. 1c), summarized as stroma. 
a

Carcinogenesis protocol 4-Nitroquinoline 1-oxide

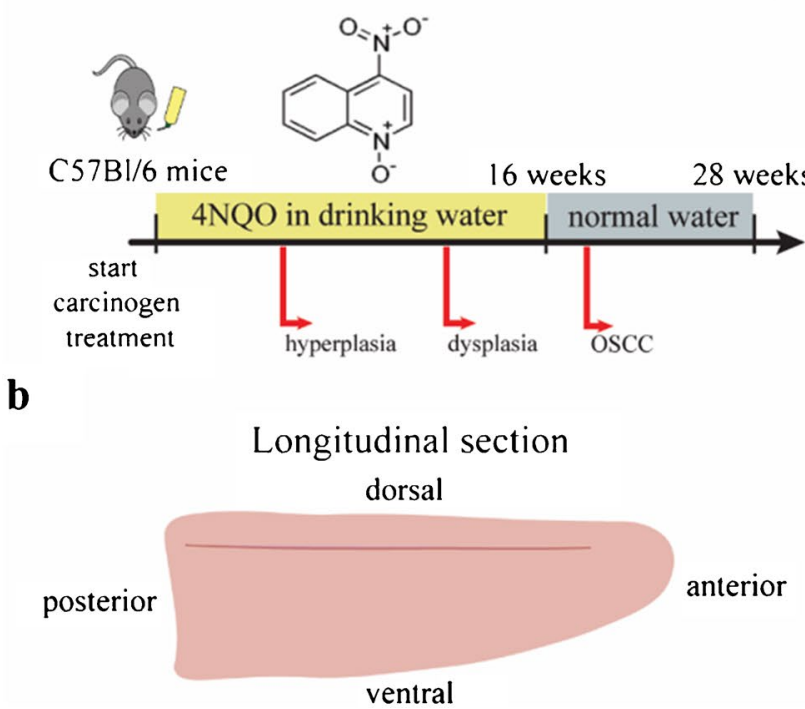

Fig. 1 Principle of tumor induction in C57B1/6 N mice. (a). At the beginning of carcinogen treatment, mice were administered the tumorigenic compound $4 \mathrm{NQO}$ via the drinking water over a total interval of 16 weeks (a). Throughout this treatment, mice developed various carcinogenic stages including hyperplasia after 6 weeks, dysplasia after 12 weeks, and oral squamous cell carcinoma (OSCC) after 18 weeks. The administration of $4 \mathrm{NQO}$ was finished in week 16 and mice were supplied with normal drinking water at that point.

\section{Workflow of the HSI PCA-DA model development and validation}

To create lingual tissue classification models, consecutive working steps need to be realized, starting from mouse breeding, drug-induced carcinogenesis protocol, tissue harvesting, and tongue preparation to the final statistical evaluation of spectroscopic image data. This holistic approach is summarized in Fig. 2.

As displayed in Fig. 2, C57B1/6 N mice were bred for a total of 28 weeks and treated with 4NQO (1). After treatment, mice tongues were harvested and a sequential cross section cutting of the tongues in a longitudinal direction was performed (2). The tongue and microtome blade (2) are schematically shown in top view. Different profile sections of each mouse were prepared on individual goldcoated microscope slides (3). ELS DF Whiskbroom and Pushbroom imaging was accomplished on histopathologically predefined tissue regions of EP, GM, and AT (4). For Whiskbroom imaging, measuring areas were kept at $150 \mu \mathrm{m} \times 150 \mu \mathrm{m}$ whereas measuring regions for the Pushbroom imaging can differ among images. An exemplary Pushbroom imaging region of $150 \mu \mathrm{m} \times 150 \mu \mathrm{m}$ is depicted in (4). PCA-DA models were developed for both

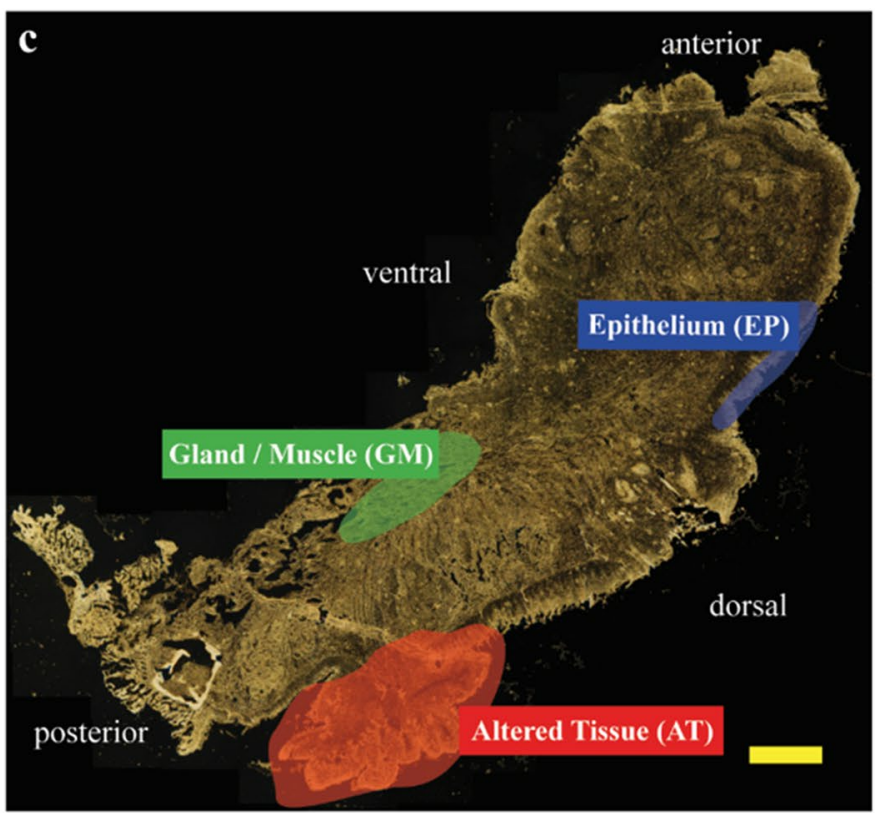

Although the 4NQO administration stopped, OSCC lesions emerged. The anatomical position and direction of longitudinal tongue cross sections is elucidated in b. An exemplary darkfield (DF) image of a mouse tongue is shown in c. Different tissue regions, such as gland/ muscle (green), epithelium (blue), and altered tissue (red), were assigned after a histopathological evaluation of a corresponding HEstained tissue section (yellow scale bar: $1000 \mu \mathrm{m}$ )

techniques in order to enable a distinct differentiation of all three tissue types (5). The Whiskbroom and Pushbroom PCA-DA models were tested by predicting modelunknown datasets of GM, EP, and AT (6). The model predictions are illustrated as colored areas for each tissue type (6).

\section{ELS spectra acquisition in Whiskbroom and Pushbroom imaging modes}

The principle of Whiskbroom and Pushbroom imaging is illustrated in Fig. 3a and b. In Whiskbroom imaging (Fig. 3a), the sample is scanned in a point-by-point manner acquiring a whole spectrum at each $\mathrm{x}, \mathrm{y}$-coordinate. In the end, the spectral image is obtained by combining all singlepoint measurements. In Pushbroom imaging (Fig. 3b), a complete line is measured at once while a spectrum at each pixel of the line is recorded. Pushbroom imaging therefore allows a much faster sample scanning compared to Whiskbroom imaging. In general, the two techniques are accompanied by varying but high spatial and spectral resolutions. Both imaging modes are applied for detecting ELS from tissue specimens within the scope of our study.

Whiskbroom images were recorded with a WITec Alpha 300 RS confocal system modified as described earlier [26] 


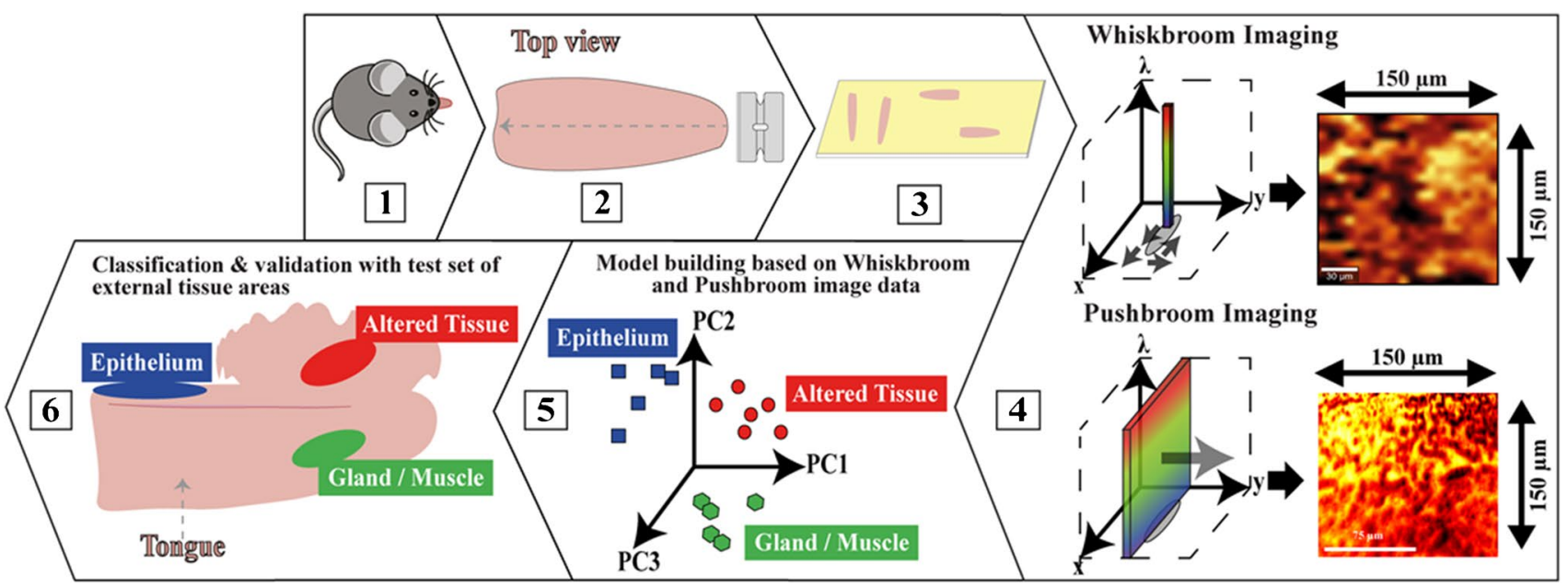

Fig. 2 Schematic illustration of the HSI PCA-DA model development and validation workflow applied for an OSCC mouse model. In 1: C57B1/6 $\mathrm{N}$ mice breeding. In 2: mice tongues harvesting and sequential cross section cutting in longitudinal direction. In 3: tongue cross sections were transferred to gold-coated microscope slides. In 4: DF ELS Whiskbroom and Pushbroom imaging of predefined tis-

(Fig. 3c). The WITec instrument was equipped with a tungsten lamp (Osram, model HLX 64625), a DF module, and a $20 \times$ DF objective (Zeiss, EC Epiplan Apochromat $20 \times / 0.6$ HD DIC M27). A $100 \mu \mathrm{m}$-core diameter multimode fiber connects the optical output to the Acton SP2300i mirror-based spectrometer fitted with an Andor DU401 DD, 35 CCD camera (EMCCD, 16 Bit, 1024×127 pixel, $26 \mu \mathrm{m} \times 26 \mu \mathrm{m}$, operating temperature: $-60^{\circ} \mathrm{C}$ ). The spectrometer was centered at $700 \mathrm{~nm}$ using a $150 \mathrm{~g} / \mathrm{mm}$ $(B L Z=800 \mathrm{~nm})$ grating. A spectral range of $412-975 \mathrm{~nm}$ was thus measurable. The complete optical setup achieves a spectral resolution of $1.6 \mathrm{~nm}$. Maximum scan areas encompass $150 \times 150 \mu \mathrm{m}$ limited by the range of the piezo table. Each spectrum was obtained with an integration time of $0.04 \mathrm{~s}$. A total number $400 \mathrm{ELS}$ spectra $(20 \times 20$ spectra $)$ was acquired in a scan area of $150 \times 150 \mu \mathrm{m}$. The applied scan steps equal to $7.5 \mu \mathrm{m}$ and the scan speed corresponds to $0.874 \mathrm{~s} /$ line. Spectralon $®$ was used as reference material measured with the same acquisition parameters. The instrumental dark current was additionally determined as dark current spectrum. Following data acquisition, ELS images were normalized with Spectralon ${ }^{\circledR}$, and dark current spectra were subtracted.

Additionally, an ELS hypercube was generated with a Zeiss MPM 800 microscope photometer implemented with a Pushbroom imaging system. The Pushbroom imager consisted of a spectrograph (Inno-Spec) and a CCD camera (QImaging, EXi Blue fluorescence microscopy camera, model: EXI-BLU-R-F-M-14-C) (Fig. 3d). Polychromatic light is generated by a tungsten lamp (Osram, model HLX 64625 ) and transferred via an optical fiber (core diameter sue regions for EP, GM, and AT. In 5: PCA-DA model development for both techniques. In 6: validation of the Whiskbroom and Pushbroom PCA-DA model with a testing set of different tissue spectra. The model predictions are illustrated as colored ellipses for each tissue type

$6.35 \mathrm{~mm}$ ) onto the tissue sample. Elastically back-scattered light was collected by the above-described $20 \times$ DF objective (Zeiss, EC Epiplan Apochromat 20×/0.6 HD DIC M27) and was finally recorded with the Pushbroom imager. The entrance slit dimension of the spectrometer corresponds to a width of $30 \mu \mathrm{m}$ and a length of $14 \mathrm{~mm}$. The spectral range encompasses 398-715 $\mathrm{nm}$ using a $600 \mathrm{~g} / \mathrm{mm}$ grating. The area of light-sensitive pixels on the Pushbroom CCD chip contains $1392 \times 1040$ pixels whereby the first pixel number depicts the spectral axis $(398-715 \mathrm{~nm})$ and the second one represents the lateral axis. Each measured line-image exhibits a spatial width of $340 \mu \mathrm{m}$ consisting of 1040 pixels. The lateral resolution is therefore 3 pixels $/ \mu \mathrm{m}$. Scan table increments were optimized to $0.3 \mu \mathrm{m}$. The number of line image scans depends on the actual tissue region and can vary between scans. The scan speed is $10 \mathrm{~s} /$ line. A spectral resolution of $1.2 \mathrm{~nm}$ can be defined for our customized setup. Comparable to the Whiskbroom data treatment, Spectralon ${ }^{\circledR}$ reference spectra as well as dark current spectra were acquired.

Corresponding excitation and detection light paths for the WITec Alpha 300 RS and the Zeiss MPM 800 microscope photometer are visualized in Fig. $3 \mathrm{c}$ and d. By implementing a DF illumination, the incident light laterally impinges on the specimen. Thus, only the diffuse ELS is detected whereas specular light is undetectable (Fig. 3c and d). Otherwise, the specular reflected light from the tissue superimposes the diffuse ELS carrying the information content and therefore hampers its detection. Due to the DF setup, no additional glare removal is necessary [36] and the diffuse ELS measurement of the sample is facilitated. Furthermore, 


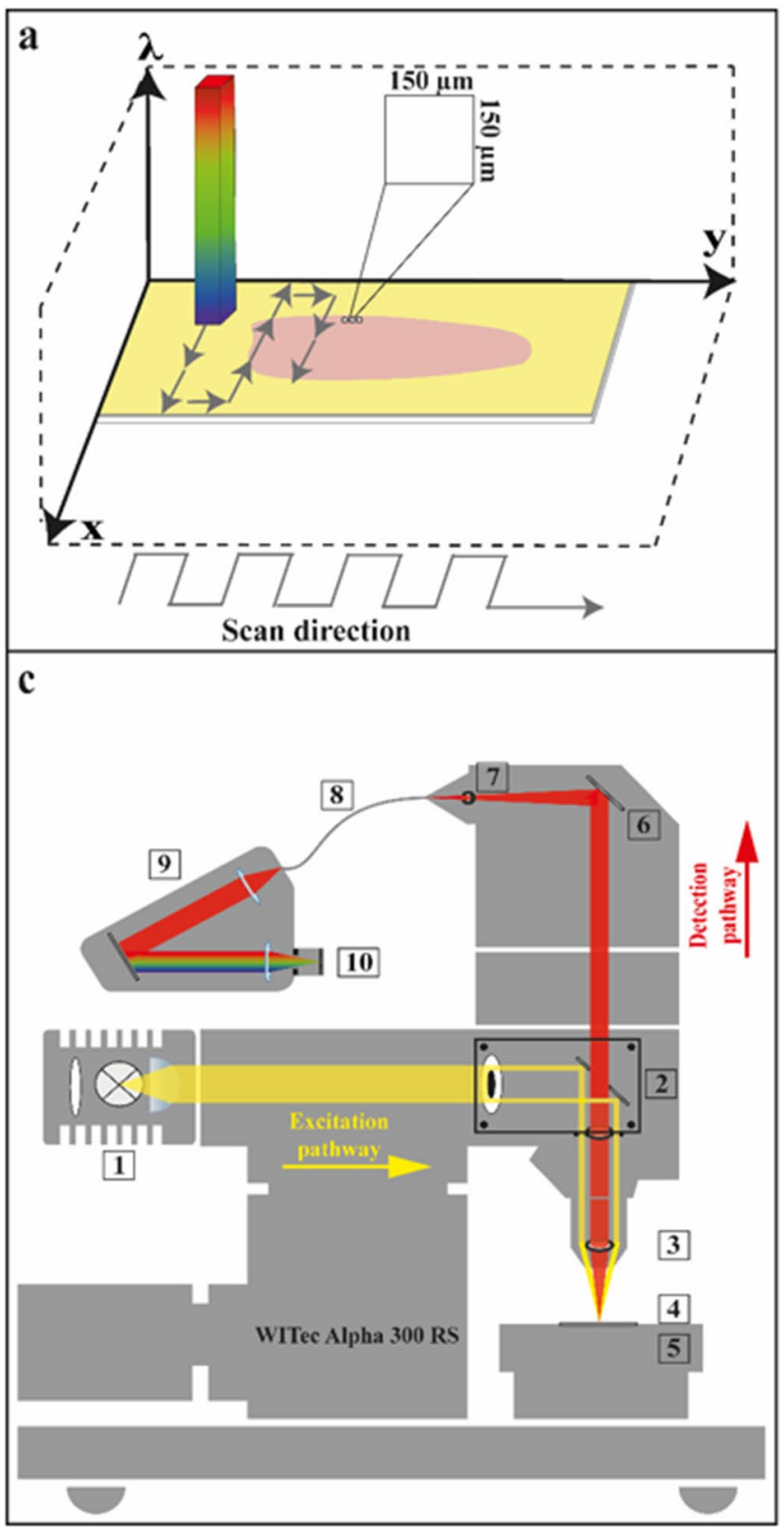

Fig. 3 Comparison of Whiskbroom and Pushbroom principles (a, b) accomplished by the two instrumental setups (c, d). In Whiskbroom imaging (a), the sample is scanned point-by-point and a whole spectrum is recorded at each $\mathrm{x}, \mathrm{y}$-coordinate. The final spectral image derives from all single-point measurements combined. Each scanned point along the tongue was performed in a step-wise manner and is equivalent to a $150 \mu \mathrm{m} \times 150 \mu \mathrm{m}$ scan size, as depicted by the zoom-in of one scan area. The small scan sizes illustrated on the mouse tongue should reveal the size proportion between scan area and tongue. In Pushbroom imaging (b), a complete line of pixels is measured simultaneously and full spectra are acquired in each pixel. The length of each scan in x-direction is fixed to $340 \mu \mathrm{m}$ whereas the movement in y-direction is variable. Applied scan ranges in y-direc-

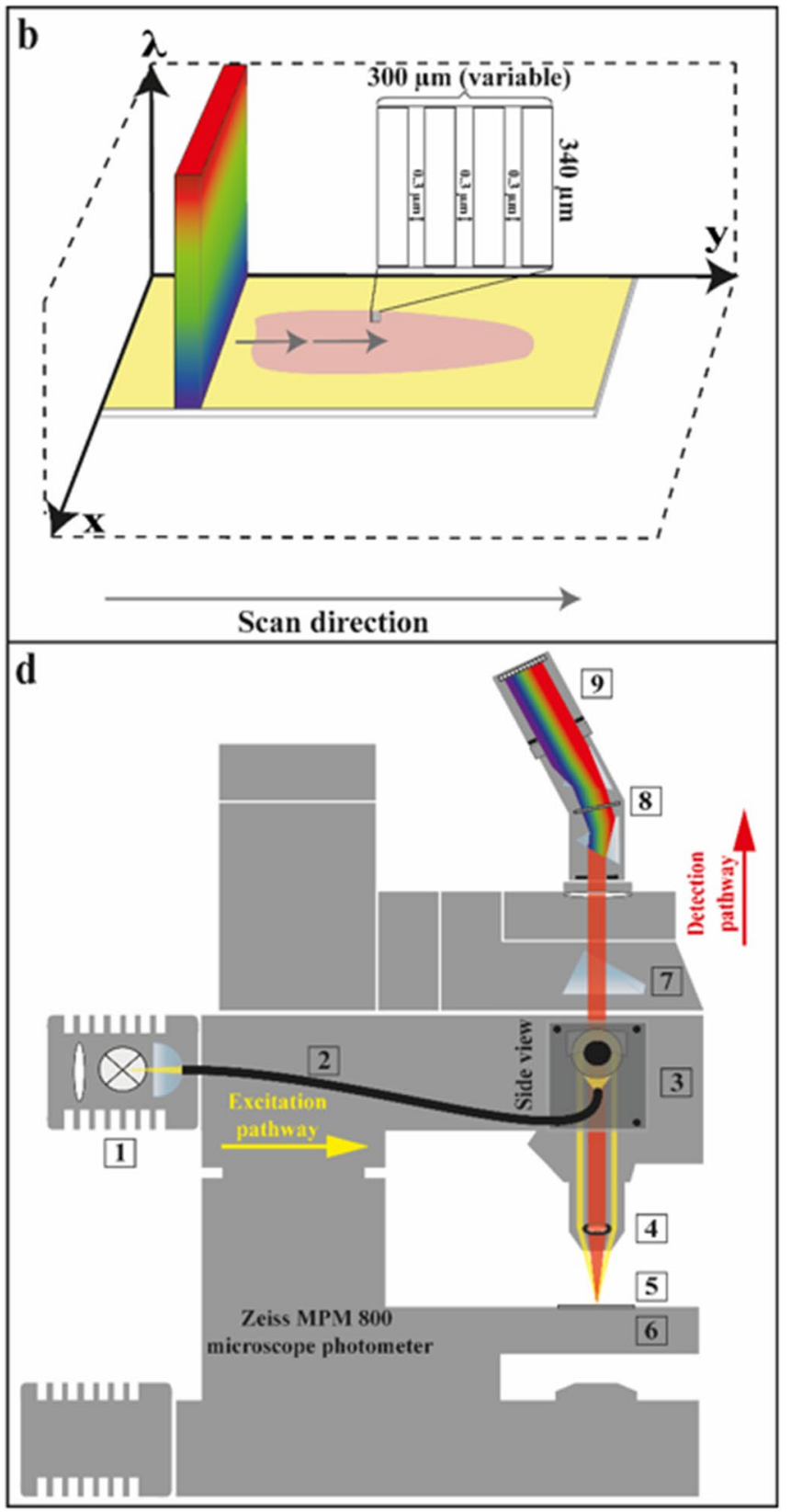

tion encompassed 180-300 $\mu \mathrm{m}$. Ideal step sizes were predetermined to be $0.3 \mu \mathrm{m}$. One image consists of the scanned $\mathrm{x}$ - and $\mathrm{y}$-direction. Whiskbroom imaging was performed by the WITec instrument (c) and Pushbroom imaging was executed by the MPM microscope photometer equipped with a Pushbroom detection system (d). WITec components are described by (c1-c10), c1: tungsten lamp, c2: darkfield module, c3: darkfield objective, c4: sample holder, c5: piezo scan table, c6: deflection mirror, c7: pinhole, c8: multimode optical fiber, c9: spectrometer, c10: CCD camera. MPM components are displayed by (d1-d9), d1: tungsten lamp, d2: optical fiber for white light transmission, d3: darkfield module (side view), d4: darkfield objective, d5: sample holder, d6: scan table, d7: prism, d8: spectrometer with optical elements, d9: imaging CCD camera 
we prepared our tissue samples on gold-coated objective slides in order to amplify the ELS of the specimen (Fig. 3a and $b$ ). Based on these adjustments, we intend to improve the overall ELS detection in order to achieve high-quality ELS data.

\section{Comparability of Whiskbroom and Pushbroom data by spatial averaging}

In order to compare Whiskbroom and Pushbroom data in a set scan area, a spatial averaging of spectra must be performed. This is due to the different lateral resolutions of both methods. In our study, a scan area size of $75 \times 75 \mu \mathrm{m}$ yields 100 ELS spectra for Whiskbroom imaging, whereas Pushbroom imaging generates 50,625 ELS spectra in the same area. By averaging 100 spectra for Whiskbroom into one spectrum and 50,625 spectra for Pushbroom imaging into a second spectrum, comparability can be ensured. These averaged spectra for both methods thus represent the identical area size of $75 \times 75 \mu \mathrm{m}$. Although DF imaging can achieve high lateral resolutions of $0.5-1 \mu \mathrm{m}$, the obtained information content is too detailed for our purpose. Therefore, we chose the above-mentioned area size of $75 \times 75 \mu \mathrm{m}$.

For the Whiskbroom PCA-DA model, a total number of $48 \mathrm{EP}, 28 \mathrm{GM}$, and 60 AT mean spectra were generated, as described above (100 spectra averaged to one spectrum for a defined area size of $75 \times 75 \mu \mathrm{m})$. The Pushbroom model was created by $33 \mathrm{EP}, 24 \mathrm{GM}$, and 44 AT mean spectra $(50,625$ spectra averaged to one spectrum for a defined area size of $75 \times 75 \mu \mathrm{m})$. For prediction purposes, a test set of spectral data was additionally measured and averaged in the previously described manner. The Pushbroom prediction data consisted of $6 \mathrm{EP}, 7 \mathrm{GM}$, and 22 AT average spectra. On the contrary, Whiskbroom data yielded 4 EP, 4 GM, and 16 AT average spectra.

\section{Data preprocessing and PCA-DA model development}

For PCA-DA model formation and testing, the software The Unscrambler ${ }^{\circledR}$ X (Camo Software, Version: 10.5) was used. Spectra of both acquisition methods were preprocessed equally with the software. At first, ELS image spectra were displayed as absorption spectra $(-\log (\mathrm{R}))$. A spectral smoothing according to Moving Average with 47 segment points was applied. Next, a baseline offset correction was performed and followed by a gap derivation $\left(1^{\text {st }}\right.$ derivative, gap size: 15 pts.). All spectra were conclusively range-normalized.

A PCA for Whiskbroom and Pushbroom spectra was calculated with mean-centering using the NIPALS-algorithm and the Leverage correction method for validation purposes. A total number of four principal components
(PC) was required to represent the spectral data. Model outliers were displayed by the influence plot illustrating the $F$-residuals vs. the Hotelling's $T^{2}$ statistic with a critical limit of $5 \%$. Spectral outliers were manually verified and removed from the model if proven to be true. Outlier spectra mostly appeared to be noisy background spectra measured in tissue holes. In combination with the PCA, a DA was accomplished with a quadratic distance calculation using four PCs.

Several validation parameters like sensitivity, specificity, and precision were calculated for both PCA-DA models in accordance with the confusion matrix terminology. They enabled a characterization of the models and demonstrated their functionality. All three parameters were computed as following

Sensitivity $=\frac{\text { True Positives }}{\text { True Positives }+ \text { False Negatives }}$

Specificity $=\frac{\text { True Negatives }}{\text { True Negatives }+ \text { False Positives }}$

Precision $=\frac{\text { True Positives }}{\text { True Positives }+ \text { False Positives }}$

Each validation parameter was weighted considering the number of ELS tissue spectra, which contribute to the model.

The graphical representation of spectral and statistical data is executed with OriginPro 2018G (OriginLab Corporation).

\section{Results}

\section{Histology of murine tongue tissues}

The identification of different tissue types within a tongue tissue section requires a histological assessment, commonly performed using HE-staining. For our study, various tissue regions of longitudinal-cut murine tongue cross sections were HE-stained and histologically classified by our pathologist Marion Roeßler. Based on the pathologist's evaluation, two distinct healthy tissue areas could be distinguished in all mice, from which epithelium and stroma could be assigned. Epithelial tissue defines the outermost layer of the tongue lining the entire organ from the ventral to the dorsal side (Fig. 4a). The epithelial tissue consists of several cell layers, which are a highly proliferative basal region, followed by a dense and metabolically highly active suprabasal layer and finally a keratinized layer on top that forms the filiform papillae of the dorsal tongue (Fig. 4a1, dashed area). Due to the layered structure of the epithelium, this tissue is highly 
heterogeneous from a cross-sectional point of view. The epithelium sits on a stromal region that consists of fibroblasts, immune cells, and small capillaries in conjunction with extracellular matrix. Adjacent to the epithelial stroma, a mixed-tissue of gland and muscle, the stroma, affiliates (Fig. 4a1). Muscle fibers run along the cross-sectional middle part of the tongue in a highly defined and structured manner (Fig. 4b1). In-between those muscle fibers, glandular tissue is embedded, but mainly located beneath the epithelial layer (Fig. 4b and b1). Carcinogen-treated mice additionally display morphological or cell-structural alterations of epithelial tissue with several tumor stages defined as hyperplasia, dysplasia, or SCC. An invasive SCC has been characterized in our mouse tongues originating from healthy epithelium after carcinogen treatment (Fig. 4b2, b3). Early epithelial changes include thickened epithelium and hyperkeratosis, followed by cellular and nuclear pleomorphism with abnormal cellular size and shape changes, nuclear hyperchromatism, and increased and abnormal mitotic figures. Moderate dysplasia presents loss of cell polarity, disordered maturation from basal to squamous cells, and increased cellular density.
Invasive SCCs grow into underlying tissue areas by disrupting the basal membrane and loss of epithelial stratification (Fig. 4b2). In some specimens, SCCs also show invasion into gland and muscle layers (Fig. 4b). One SCC tissue segment could even be specified as a carcinoma variation with an adenocarcinoma part (Fig. 4b3) [37]. Representative areas of healthy and altered tissue regions were chosen for DF ELS imaging according to the pathologist's evaluation.

\section{Whiskbroom PCA-DA model}

All tissue type spectra were area-averaged, as described above, to a final number of $48 \mathrm{EP}, 28 \mathrm{GM}$, and $60 \mathrm{AT}$ mean spectra used for the PCA-DA model formation ("Materials and methods"). The used spectra originated from three different mice (mouse A-C). In Fig. 5a, three exemplary Whiskbroom ELS spectra of EP, GM, and AT without any data preprocessing steps are shown. The ELS spectra are dominated by a wave-like appearance superimposed by even finer scattering patterns [38]. These typical features are not always directly visible in ELS spectra since the diffuse

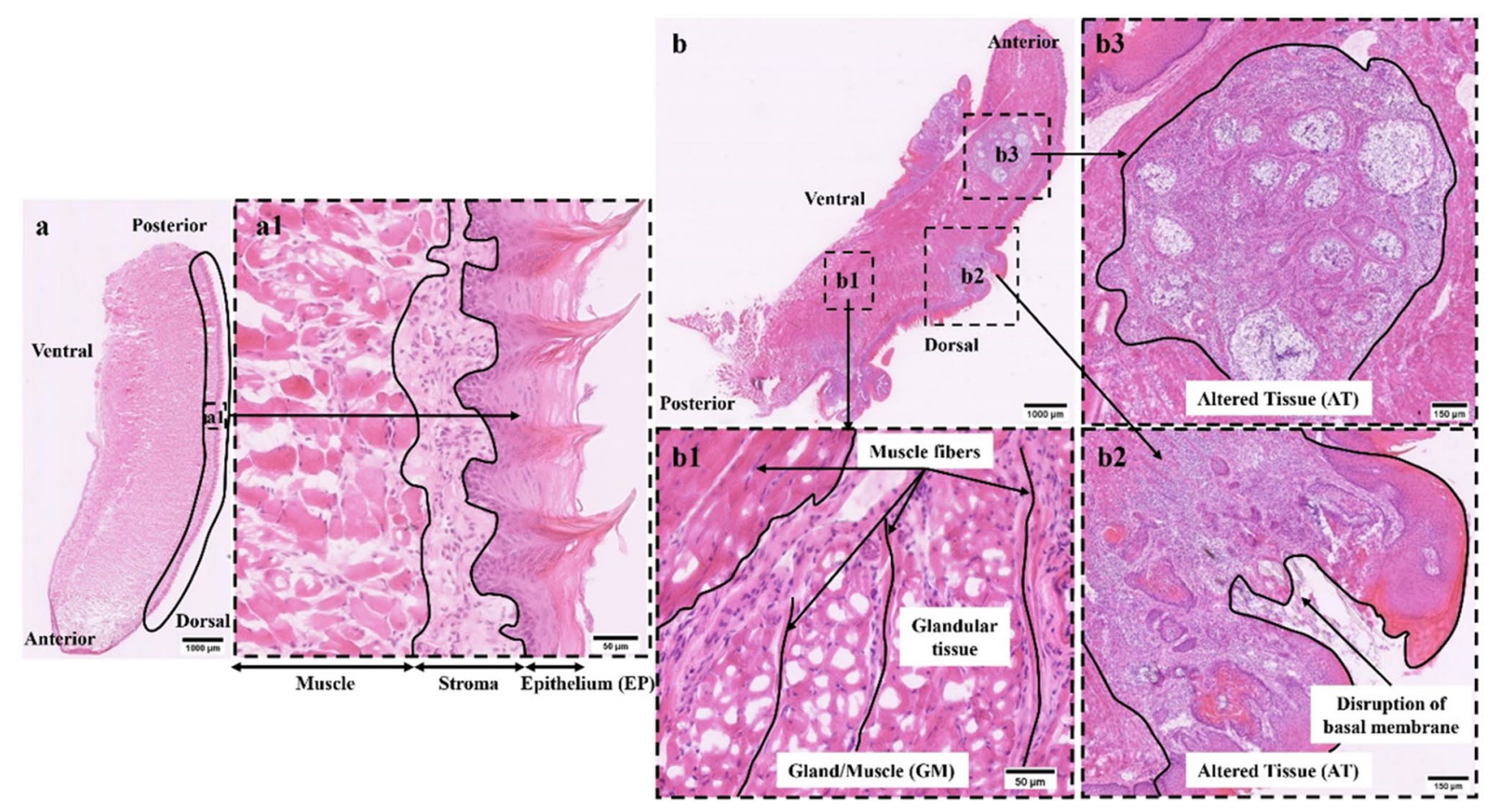

Fig. 4 Histological description of representative tissue regions for epithelium (EP), gland/muscle (GM), and altered tissue (AT). A longitudinal tongue section of mouse a with its anatomical orientation is shown in a. Epithelium in dorsal direction is marked by the black frame (a). An enlarged view of the dorsal epithelium (a1, dotted line) points out its multilayered structure. The keratinized top layer merges into a metabolically active epithelium layer allying to the stroma. The metabolic active epithelium is separated from the stroma by the basement membrane. Within the uppermost layer, filiform papillae are embedded in a tip shape. Typical gland/muscle (b1, dotted line) and OSCC tissue areas (b2, b3, dotted line) are located in an overview tissue section of mouse $\mathrm{C}(\mathrm{b})$. Glandular tissue is mainly implemented into muscle tissue indicated by the vertically oriented muscle fibers (b1). OSCCs are visible in b2 and b3. OSCC undergoes epithelialmesenchymal transition, invading subjacent tissues and interrupts the highly organized epithelium layer (b2). One OSCC branch additionally exhibits adenocarcinoma parts (b3) 
Fig. 5 PCA model based on Whiskbroom data for the differentiation of EP (blue), GM (green), and AT (red). In a: exemplary ELS spectra of EP (blue), GM (green), and AT (red) tissue are depicted without any data preprocessing steps. They are displayed as absorption spectra $(-\log (\mathrm{R}))$. In $\mathrm{b}$ : the 3D scores plot of the PCA reveals the separation of GM, EP, and AT groups by PC1, PC2, and PC4 explaining $85 \%, 5 \%$, and $1 \%$ of the overall variance. It shows the importance of a third PC to achieve a distinctly improved segregation. Only preprocessed ELS spectra are considered for the PCA calculation. Preprocessing steps encompass: moving average smoothing with 47 segment points, baseline offset correction, gap derivation $\left(1^{\text {st }}\right.$ derivative, gap size: 15 pts.), and range-normalization. In c: corresponding loadings plots for each PC of the calculated PCA. In Supplementary Material: the 2D scores plot of PC4 against PC3 as well as PC3 against PC2 with the related loadings plots are shown (Fig. S2)

scattering intensity is often weak and the spectral shape is dominated by the gold substrate (Fig. 5a). The y-axial intensity displacement among EP, GM, and AT spectra can mainly be ascribed to influences of different slice thicknesses (Fig. 5a). Thus, data preprocessing is mandatory to extract the hidden scattering information from the ELS spectra and to remove unwanted effects. The Whiskbroom PCA-DA calculation was only accomplished with preprocessed ELS spectra ("Materials and methods"). Exemplary preprocessed spectra are illustrated in Supplementary Material, Fig. S1a.

The resulting PCA for ELS Whiskbroom spectra is illustrated in Fig. 5b and c. The 3D scores plot shows PC1, PC2, and PC4 representing $85 \%$ for $\mathrm{PC} 1,5 \%$ for $\mathrm{PC} 2$, and $1 \%$ for PC4 of the total variance. All three PCs are required to achieve a separation of the investigated tissue types. Although partly overlapping, each tissue type forms one conglomeration. PC1 assigns mostly below-average score values to the spectral GM and EP clusters whereas the AT group is mainly characterized by above-average score values (Fig. 5b). In contrast to PC1, the impact of PC2 is less pronounced since it only affects a complete separation of the GM agglomeration from the AT group. It does not improve the segregation of the EP cluster. The EP group lies diagonally in-between GM and AT within the PC2 vs. PC1 plain of the scores plot (Fig. 5b). Overall, PC2 promotes a slanted differentiation of the three tissue-type clusters. Corresponding loadings plots of $\mathrm{PC} 1$ and $\mathrm{PC} 2$ reveal the greatest effects on the cluster formation in a wavelength region of 400-600 nm with two loadings maxima at $430 \mathrm{~nm}$ and $503 \mathrm{~nm}$ for PC1 and one major negative maximum at $540 \mathrm{~nm}$ for PC2 (Fig. 5c, dashed and dotted line). Above $600 \mathrm{~nm}$, a poorly distinct ripple structure is visible for both loadings plots. The loadings curves demonstrate the effect of the spectral ELS signature on the tissue cluster separation. PC4 finally enables a significantly improved separation of all three tissue agglomerations (Fig. 5b). The main influence of PC4 is the differentiation of GM and EP spectra from one another and it more precisely defines and shapes the tissue clusters. Examination of the PC4 loadings plot shows a
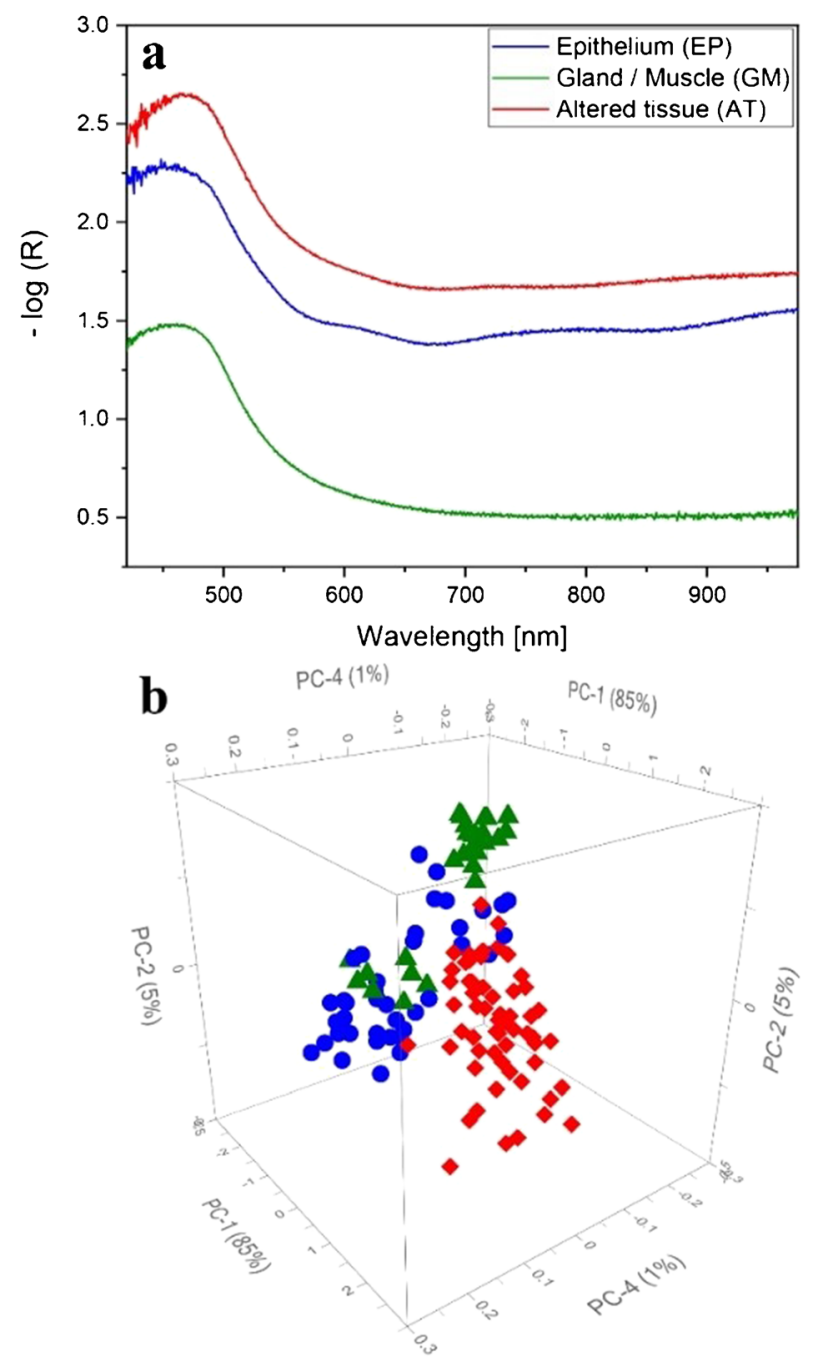

- Epithelium (EP) A Gland / Muscle (GM)

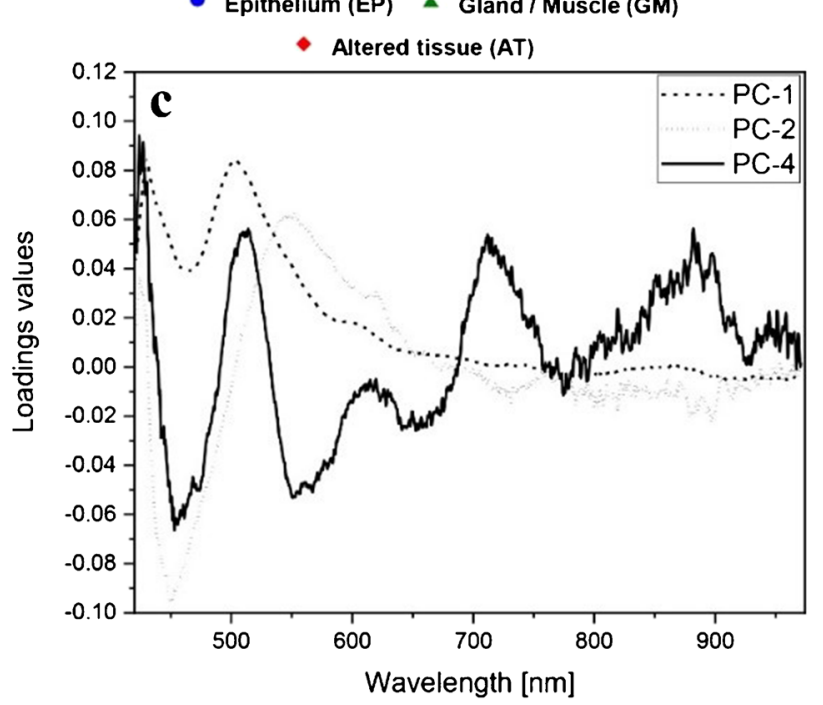

repeatedly occurring pattern partly overlaid by even smaller ripple elements, which refer to Mie scattering (Fig. 5c, solid 
line). The segregation of the tissue clusters is dominated by these effects.

Based on the PCA calculation, a DA was subsequently computed and combined to a final PCA-DA model that allows the classification of ELS spectra from unknown lingual tissue areas. The actual model was created by a training set of ELS spectra for EP, GM and AT and optimized with respect to best classification results. By applying the DA, a validation of the used quadratic distance algorithm with 4 PCs was performed and illustrated as a confusion matrix (s. Supplementary Material, Tab. S1). As part of the confusion matrix results, the training spectra themselves were predicted by the algorithm and afterwards grouped dependent on the prediction outcome. An overview of the training spectra prediction is summarized in Table 2. Except for the AT group, the DA algorithm correctly predicted all EP and GM training sets (Table 2). The overall model accuracy corresponds to $98 \%$ for the Whiskbroom data in total.

For a precise characterization of the PCA-DA, further model parameters such as sensitivity, specificity, and precision were determined to represent the model's performance. The parameters were calculated from the confusion matrix (s. Supplementary Material, Tab. S1) and describe the model quality. Dependent on the amount of ELS training data for each tissue type, the training spectra contribute differently to the calculation of the respective model parameters. This contribution was considered in terms of different weightings for the tissue groups. A summary of the weighted sensitivity, specificity, and precision is presented in Table 2. The Whiskbroom model shows holistic sensitivity and precision values of $98 \%$ and even exhibits a specificity of $99 \%$. These parameters demonstrate that the model was optimized as effectively as possible with the training set. Thus, reasonable classifications of unknown ELS spectra are expected.

\section{Pushbroom PCA-DA model}

For the Pushbroom PCA-DA model, 33 EP, 24 GM, and 44 AT spectra were area-averaged in the above-described manner ("Materials and methods"). All spectra were obtained from three different mice (mouse A-C). Exemplary Pushbroom spectra of each tissue type without any data preprocessing steps are illustrated in Fig. 6a. As mentioned above, ELS spectra show a typical sinusoidal shape overlaid by smaller scattering patterns [38] which is hardly visible in the Pushbroom spectra (Fig. 6a). Therefore, a data preprocessing is again necessary to gain the actual scattering information and remove measuring- or sample-ascribable effects, such as the y-axial intensity displacement due to varying slice thicknesses or the impact of the gold substrate on the overall spectral trend (Fig. 6A). The Pushbroom PCA-DA calculation was only accomplished with preprocessed ELS spectra ("Materials and methods"). Exemplary preprocessed spectra are illustrated in the Supplementary Material, Fig. S1b.

The PCA for the ELS Pushbroom spectra is displayed in Fig. $6 \mathrm{~b}$ and c. Three PCs are required to achieve an almost complete segregation of the EP (blue), GM (green), and AT clusters (red). The 3D scores plot shows PC1, PC2, and PC4 representing $61 \%$ for $\mathrm{PC} 1,19 \%$ for $\mathrm{PC} 2$, and $1 \%$ for PC4 of the total variance. Due to PC1 and PC2, the tissue clusters are arranged around the scores plot center with only minor overlapping of all three groups. PC1 mainly separates the GM and EP tissue clusters from the AT conglomeration. Since GM and EP are mostly defined by below-average score values and AT by above-average ones, all groups are organized in the described manner. PC2, however, primarily enables a segregation of the EP group from the GM and AT clusters with a partial overlay of the conglomerations. The related loadings plots for PC1 (Fig. 6c, dashed line) and PC2 (Fig. 6c, dotted line) explain the arrangements of the three tissue groups in the scores plot based on dominating spectral effects. Within the loadings plot for PC1 (Fig. 6c, dashed line), two spectral maxima at $411 \mathrm{~nm}$ and $495 \mathrm{~nm}$ are observable. The loadings plot for PC2, however, shows one main negative peak at $420 \mathrm{~nm}$. For both, a periodic spectral course is implied in a higher wavelength range of 550-700 nm. PC4 is essential to achieve an almost complete differentiation of the EP, GM and AT aggregation (Fig. 6b). The scores plot (Fig. 6b) demonstrates that PC4 not only

and in percentage terms (columns 3 and 4). The last columns (columns 5-8) represent the overall accuracy, sensitivity, specificity, and precision of the PCA-DA model
Table 2 Model-related quality parameters of the Whiskbroom PCADA. In addition to the total number of training spectra (column 2), their prediction results by the DA are listed as absolute numbers

\begin{tabular}{|c|c|c|c|c|c|c|c|}
\hline Tissue type & $\begin{array}{l}\text { Total spectra } \\
\text { in model }\end{array}$ & $\begin{array}{l}\text { Correctly predicted } \\
\text { model spectra }\end{array}$ & $\begin{array}{l}\text { Proportion of correctly pre- } \\
\text { dicted model spectra [\%] }\end{array}$ & $\begin{array}{l}\text { Accuracy } \\
{[\%]}\end{array}$ & $\begin{array}{l}\text { Sensitivity } \\
{[\%]}\end{array}$ & $\begin{array}{l}\text { Specificity } \\
{[\%]}\end{array}$ & $\begin{array}{l}\text { Precision } \\
{[\%]}\end{array}$ \\
\hline $\begin{array}{l}\text { Gland/muscle } \\
\text { (GM) }\end{array}$ & 28 & 28 & 100 & 98 & 98 & 99 & 98 \\
\hline $\begin{array}{l}\text { Epithelium } \\
\text { (EP) }\end{array}$ & 41 & 41 & 100 & & & & \\
\hline $\begin{array}{l}\text { Altered tissue } \\
\text { (AT) }\end{array}$ & 58 & 56 & 97 & & & & \\
\hline
\end{tabular}


Fig. 6 PCA model based on Pushbroom data for the differentiation of EP (blue), GM (green), and AT (red). In a: exemplary ELS spectra of EP (blue), GM (green), and AT (red) are depicted without any data preprocessing steps. They are displayed as absorption spectra $(-\log (\mathrm{R}))$. In $\mathrm{b}$ : the 3D scores plot of the PCA reveals the separation of GM, EP, and AT groups by PC1, PC2, and PC4 explaining 61\%, $19 \%$, and $4 \%$ of the overall variance. It shows the importance of a third PC to achieve a distinctly improved segregation. Only preprocessed ELS spectra are considered for the PCA calculation. Preprocessing steps encompass: moving average smoothing with 47 segment points, baseline offset correction, gap derivation $\left(1^{\text {st }}\right.$ derivative, gap size: 15 pts.), and range-normalization. In c: corresponding loadings plots for each PC of the calculated PCA. In Supplementary Material: the 2D scores plot of PC4 against PC3 as well as PC3 against PC2 with the related loadings plots are shown (Fig. S3)

accomplishes a total segregation of the AT cluster from EP and GM, but also further separates the EP group from the GM one. Nevertheless, a slight overlapping between the EP and GM clusters remains. The examination of the PC4 loadings plot (Fig. 6c, solid line) reveals negative maxima at $411 \mathrm{~nm}$ and $450 \mathrm{~nm}$ as well as a distinct spectral ripple pattern dominating above $530 \mathrm{~nm}$ which refer to Mie scattering (Fig. 6c, solid line).

Subsequently, a DA was calculated, as already described for the Whiskbroom model. By assigning the ELS training spectra with the used DA algorithm, the model performance was verified. The performance results were summarized in a confusion matrix (s. Supplementary Material, Tab. S2). As the majority of training spectra were correctly matched by the model, an overall accuracy of $98 \%$ was achieved (Table 2). All investigated AT spectra were entirely dedicated to AT, whereas $32 \mathrm{EP}$ and $23 \mathrm{GM}$ spectra out of $33 \mathrm{EPs}$ and $24 \mathrm{GMs}$ were also attributed to EP and GM, respectively.

Identical model parameters were also calculated to characterize the model more accurately (Table 3). They indicate the model's applicability for classification purposes. All parameters were again calculated as weighted values that consider the different quantities of EP, GM, and AT spectra in the training set (Table 3). Results for the weighted accuracy, sensitivity, specificity, and precision were comparable to the Whiskbroom model.

\section{Classification of tissue types by PCA-DA models}

Our classification approach is visualized in Fig. 7. At first, a histopathological identification of all three tissue types by HE-stained tissue sections had to be performed in order to localize the tissues on the ELS-ready tissue sections (Fig. 7a and b). Based on this tissue assignment, ELS test spectra of the tissue types were acquired and finally classified by the PCA-DA model (Fig. 7b, white crosshairs). To validate whether the classification of the model is true, the classification results were compared with the HE diagnosis of the exact same tissue regions. As indicated
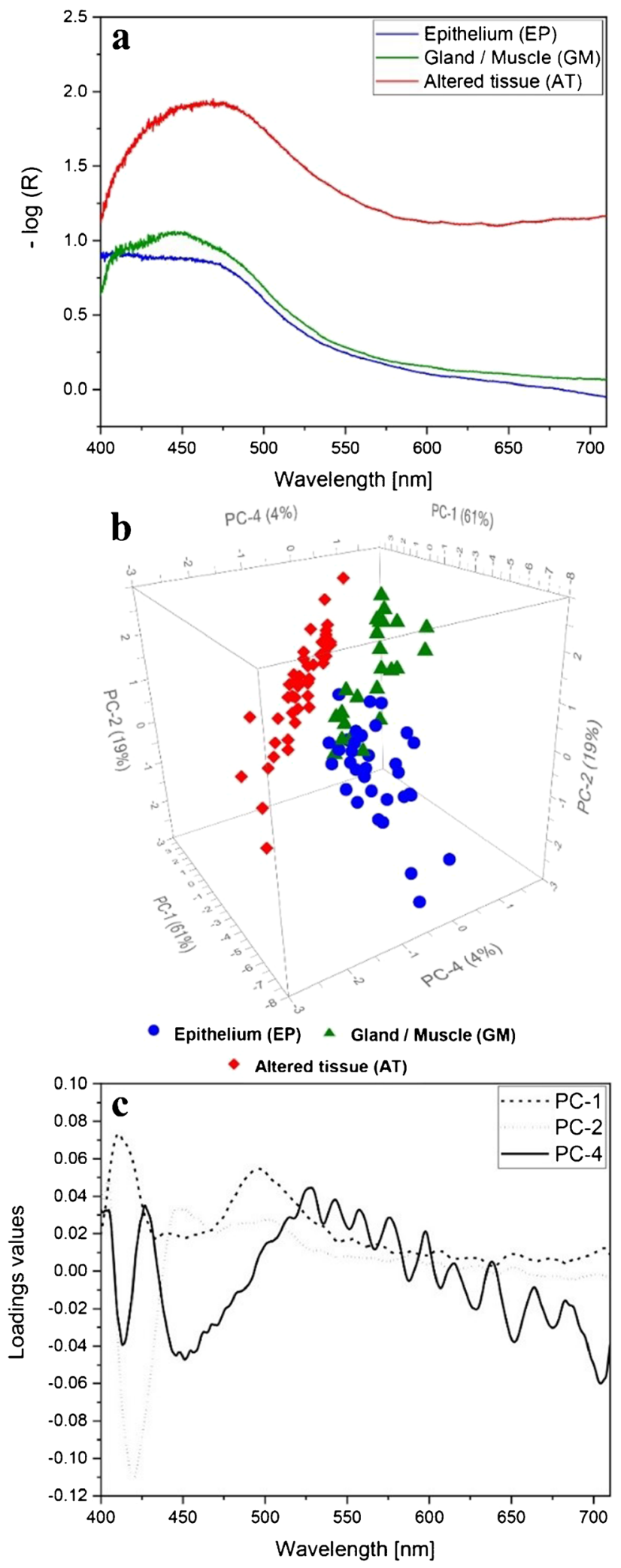

by the white crosses or checkmarks (Fig. 7c), the prediction by the model either conforms to the HE-identification 
Table 3 Model-related quality parameters of the Pushbroom PCADA. In addition to the total number of ELS training spectra (column 2), the classification results of the training set were listed as absolute numbers and in percentage terms (columns 3 and 4). The last columns (columns 5-8) represent the overall accuracy, sensitivity, specificity, and precision of the PCA-DA

\begin{tabular}{|c|c|c|c|c|c|c|c|}
\hline Tissue type & $\begin{array}{l}\text { Total spectra } \\
\text { in model }\end{array}$ & $\begin{array}{l}\text { Correctly predicted } \\
\text { model spectra }\end{array}$ & $\begin{array}{l}\text { Proportion of correctly pre- } \\
\text { dicted model spectra [\%] }\end{array}$ & $\begin{array}{l}\text { Accuracy } \\
{[\%]}\end{array}$ & $\begin{array}{l}\text { Sensitivity } \\
{[\%]}\end{array}$ & $\begin{array}{l}\text { Specificity } \\
{[\%]}\end{array}$ & $\begin{array}{l}\text { Precision } \\
{[\%]}\end{array}$ \\
\hline $\begin{array}{l}\text { Gland/muscle } \\
\text { (GM) }\end{array}$ & 24 & 23 & 96 & 98 & 98 & 99 & 98 \\
\hline $\begin{array}{l}\text { Epithelium } \\
\text { (EP) }\end{array}$ & 33 & 32 & 97 & & & & \\
\hline $\begin{array}{l}\text { Altered tissue } \\
\text { (AT) }\end{array}$ & 44 & 44 & 100 & & & & \\
\hline
\end{tabular}
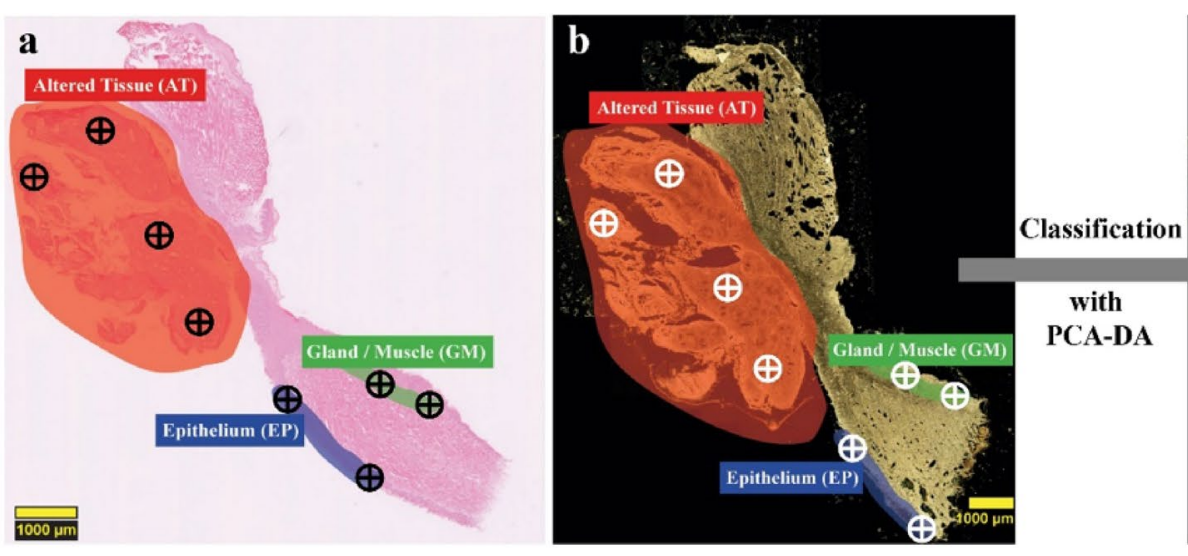

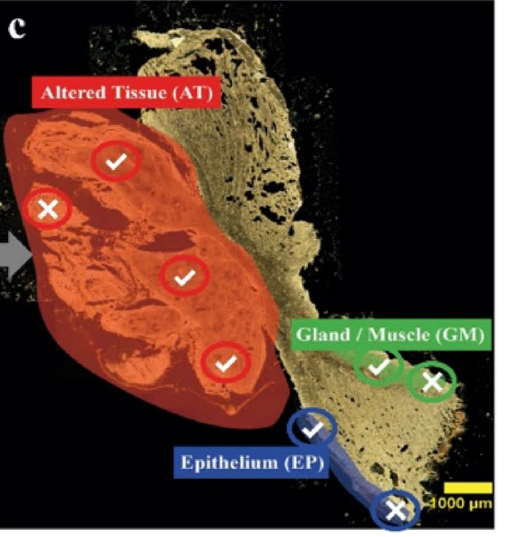

Fig. 7 Classification principle of the PCA-DA models. In a: a HEbased identification of different tissue types from a to-predict tissue section. The crosshairs in a indicate the tissue spots, which need to be located in $b$ for ELS spectra measurements. In b: localization of the same tissue regions and spots on corresponding tissue sections used for ELS data acquisition. According to this localization, ELS

(checkmark) or differed from it (cross). The corresponding prediction results are summarized in Table 4.

For both PCA-DA models, the test set of EP, GM, and AT spectra was obtained as described earlier ("Materials and methods"). Since the classification models were created by ELS training spectra of three different mouse samples (mouse A-C), the tissue of a fourth mouse (mouse D) was considered for testing the AT classification. The additional mouse sample should emphasize that the model can correctly assign unknown AT spectra of a completely new tissue sample. Furthermore, unknown EP and GM tissue regions of mouse samples A, B, and C were also examined with the PCA-DA models.

A classification of 12 different AT regions of mouse $\mathrm{D}$, four EP regions, and two GM areas of mouse $\mathrm{C}$ was performed by the Whiskbroom model. A test set of 6 different EP spectra of mouse A, 7 GM spectra of mouse $\mathrm{B}$, and 22 AT spectra of mouse D, however, was used to verify the classification abilities of the Pushbroom model. A summary of the model classification results test spectra for the different tissue types were measured at each spot (white crosshairs) and were afterwards classified by the PCA-DA models. In c: as implied by the white crosses or checkmarks, the prediction of the ELS test spectra by the model either coincides with the HE identification (checkmark) or differs from it (cross)

and the alignment with the HE diagnosis is shown in Table 4. Since the PCA-DA models were mainly developed for the AT recognition in tongues, mostly AT tissues were included in the testing set. Overall, our classification procedure reveals the true prediction capability of our models.

\section{Discussion}

In this proof-of-concept study, we compare Whiskbroom and Pushbroom DF ELSS imaging and apply both to a HNSCC mouse trial. ELS image data of murine lingual cross sections were acquired and subsequently pre-processed to eliminate recording-related influences. Afterwards, the ELS data were subjected to a PCA to allow a data reduction and extraction of the important tissues scattering information. By combining the PCA-structured ELS data with a DA, a Whiskbroom and Pushbroom PCADA model was formed. These models were used to classify 
Table 4 Summary of the PCA-DA model classification results and HE-diagnosed conclusions. ELS test spectra of different tissue regions for AT, $\mathrm{EP}$, and GM were classified by both models and HE-evaluated by a blinded pathologist. Epithelium regions (1-4) of the Whiskbroom model adjoin to one of the AT areas and are thus declared as EP margin. If the model classification and the $\mathrm{HE}$ diagnosis coincide, they are marked in green. Otherwise, they are highlighted in red and bold

\begin{tabular}{|c|c|c|c|c|c|c|c|}
\hline \multicolumn{4}{|c|}{ Whiskbroom classification } & \multicolumn{4}{|c|}{ Pushbroom classification } \\
\hline $\begin{array}{l}\text { Defined } \\
\text { tissue type }\end{array}$ & $\begin{array}{l}\text { Prediction } \\
\text { region }\end{array}$ & $\begin{array}{l}\text { Model } \\
\text { classified } \\
\text { as }\end{array}$ & $\begin{array}{c}\text { HE } \\
\text { diagnosed } \\
\text { as }\end{array}$ & $\begin{array}{l}\text { Defined } \\
\text { tissue type }\end{array}$ & $\begin{array}{l}\text { Prediction } \\
\text { region }\end{array}$ & $\begin{array}{l}\text { Model } \\
\text { classified } \\
\text { as }\end{array}$ & $\begin{array}{c}\text { HE } \\
\text { diagnosed } \\
\text { as }\end{array}$ \\
\hline \multirow{12}{*}{$\begin{array}{l}\text { Altered tissue } \\
\text { (AT) }\end{array}$} & 1 & EP & AT & \multirow{22}{*}{$\begin{array}{l}\text { Altered tissue } \\
\text { (AT) }\end{array}$} & 1 & EP & $\mathrm{AT}$ \\
\hline & 2 & $\overline{\mathrm{AT}}$ & AT & & 2 & GM & AT \\
\hline & 3 & EP & AT & & 3 & AT & AT \\
\hline & 4 & EP & AT & & 4 & $\mathrm{AT}$ & AT \\
\hline & 5 & AT & AT & & 5 & AT & AT \\
\hline & 6 & AT & AT & & 6 & AT & AT \\
\hline & 7 & $\mathrm{AT}$ & $\mathrm{AT}$ & & 7 & $\mathrm{AT}$ & AT \\
\hline & 8 & AT & AT & & 8 & AT & AT \\
\hline & 9 & AT & AT & & 9 & AT & AT \\
\hline & 10 & AT & AT & & 10 & AT & AT \\
\hline & 11 & AT & $\mathrm{AT}$ & & 11 & $\mathrm{AT}$ & $\mathrm{AT}$ \\
\hline & 12 & EP & AT & & 12 & $\mathrm{AT}$ & AT \\
\hline \multirow{4}{*}{$\begin{array}{c}\text { Epithelium } \\
\text { (margin) } \\
(\mathrm{EP})\end{array}$} & 1 & EP & AT & & 13 & $\mathrm{AT}$ & $\mathrm{AT}$ \\
\hline & 2 & $\mathrm{AT}$ & $\mathrm{AT}$ & & 14 & AT & AT \\
\hline & 3 & $\mathrm{AT}$ & AT & & 15 & $\mathrm{AT}$ & $\mathrm{AT}$ \\
\hline & 4 & AT & AT & & 16 & AT & AT \\
\hline \multirow{19}{*}{$\begin{array}{c}\text { Gland/Muscle } \\
(\mathrm{GM})\end{array}$} & 1 & GM & $\overline{\text { GM }}$ & & 17 & AT & $\mathrm{AT}$ \\
\hline & 2 & GM & GM & & 18 & $\mathrm{AT}$ & AT \\
\hline & & & & & 19 & AT & AT \\
\hline & & & & & 20 & AT & AT \\
\hline & & & & & 21 & AT & AT \\
\hline & & & & & 22 & AT & AT \\
\hline & & & & \multirow{6}{*}{$\begin{array}{l}\text { Epithelium } \\
\text { (EP) }\end{array}$} & 1 & EP & EP \\
\hline & & & & & 2 & GM & EP \\
\hline & & & & & 3 & EP & EP \\
\hline & & & & & 4 & EP & EP \\
\hline & & & & & 5 & GM & EP \\
\hline & & & & & 6 & EP & EP \\
\hline & & & & \multirow{7}{*}{$\begin{array}{c}\text { Gland/Muscle } \\
\text { (GM) }\end{array}$} & 1 & $\overline{\text { GM }}$ & $\overline{\text { GM }}$ \\
\hline & & & & & 2 & GM & GM \\
\hline & & & & & 3 & GM & GM \\
\hline & & & & & 4 & GM & GM \\
\hline & & & & & 5 & GM & GM \\
\hline & & & & & 6 & GM & GM \\
\hline & & & & & 7 & GM & GM \\
\hline
\end{tabular}

unknown mouse tissue spectra and to distinguish between different tongue tissue types. A verification of the PCA-DA models was performed with an external set of ELS test spectra. Identical tissue regions were histologically evaluated and compared to the models' classification results for validation purposes. This comparison finally allows determining if one of the PCA-DA models is more suitable to correctly identify the investigated tissue state. To our knowledge, the comparison of two different DF ELSS HSI detection principles in terms of tissue identification was conducted for the first time. So far, HSI only coupled with other spectral techniques was applied for the differentiation of healthy and cancerous tissue, such as Raman [39], fluorescence [40], or FT-IR imaging [25].

The application of a PCA is mandatory for the enormous amount of data recorded by Whiskbroom and Pushbroom imaging. The PCA is an objective and unbiased analyzing tool [41], which helps to structure the ELS data matrix.
Suitable data preprocessing removes sample- and acquisition-associated effects, such as substrate background or section thickness. Due to the effects of PCA and data preprocessing, the main spectral influences for the tissue differentiation can be determined [42]. This is very important because the interpretation of ELS spectra is a demanding task as the spectral bands are not directly linked to a chemical group/vibration. The ELS patterns originate from various tissue constituents with changing refractive index, size and shape and are complicated to assign to one specific tissue component.

\section{Interpretation of Whiskbroom and Pushbroom PCA-DA models}

A combined interpretation of cluster locations within the scores plot and the corresponding loadings plots allows a deduction of spectral influences that cause the tissue group 
separation. The tissue clusters in the Pushbroom PCA seem to be slightly better separated from one another with an overall less pronounced overlay compared to the Whiskbroom PCA (Fig. 5b, Fig. 6b). Analyzing the related loadings plots of PC1 and PC2 for both PCAs (Fig. 5c, Fig. 6c) reveals great effects within a wavelength range of $400-600 \mathrm{~nm}$. Since PC1 of both PCAs separates the two healthy tissue groups from the tumor cluster, the impact of this wavelength region could be ascribed to changes in the cell nuclei of the tissues [43]. Nuclei in tumorous tissues are often enlarged and vary in size and shape compared to healthy nuclei [44]. Within the same wavelength range, the PC2 loadings plot shows a negative maximum at $450 \mathrm{~nm}$ for the Whiskbroom and at $430 \mathrm{~nm}$ for the Pushbroom PCA (Fig. 5c, Fig. 6c). In this case, this effect is mostly responsible for the cluster order along the PC2 axis. Based on the Whiskbroom cluster order, the below-average PC2 loadings maximum could give a hint to the structural organization of the three tissues. Since AT arises from intact EP, some tissue areas within the cancer region can still be healthy and thus might have the typical epithelium structure. This would explain the higher overlapping degree of the AT and EP group regarding PC2. The GM, however, is built up in a completely different manner in comparison to the EP or AT and consequently forms the most separated cluster. A small overlay of EP and GM groups according to PC2 can be explained by the close proximity of both tissues. For the Pushbroom PC2, however, the tissue group order is different. Although histologically identical tissue regions were chosen, the tissue structure and heterogeneity of the selected areas can differ compared to the Whiskbroom model and thus result in a varying cluster order. Above $600 \mathrm{~nm}$, a sinusoidal curve progression is additionally indicated in both PC1 and PC2 loadings plots (Fig. 5c, Fig. 6c). These curve shapes are presumed to result from the superposition of many scattering events. Such patterns are amplified by the underlying gold substrate because of the gold's pronounced reflectivity above $600 \mathrm{~nm}$ [45]. The application of PC4 causes an even more improved tissue cluster segregation for the Whiskbroom and Pushbroom PCA (Fig. 5b, Fig. 6b). This improvement can directly be attributed to the periodic structure that dominates throughout the entire spectral range in the PC4 loadings plots (Fig. 5c, Fig. 6c). Again, the periodic shape is expected to correlate with the scattering events related to cellular changes in the tissues [27, 38]. Alterations in cellular or subcellular units affect the sophisticated tissue structure and thus its morphology and texture [46, 47]. Differences in pattern frequency are observable in both PC4 loadings plots. The Whiskbroom PC4 loading reveals a long-wave sinusoidal shape superimposed by small ripple patterns whereas the Pushbroom loadings plot of PC4 is characterized by a ripple pattern of much higher frequency (Fig. 5c, Fig. 7c). A possible explanation can be deduced from the detection variations of both imaging setups. Since the Whiskbroom setup is a confocal scanning system, the scattering detection just takes place in the confocal volume and is thus very sensitive. Therefore, scattering of small tissue particles in addition to larger ones is accurately detectable influencing the observed pattern for the Whiskbroom PC4 loading. The high-frequency curve shape of the Pushbroom PC4 loading, however, might be correlated to the much larger detection spot of the Pushbroom setup. Therefore, no distinction of different tissue microstructures is possible and thus results in an overall scattering impression of higher frequency. Ninety-eight percent of all Whiskbroom and Pushbroom model-included spectra were accurately attributed to their corresponding tissue group (Tables 2 and 3). Additional model parameters confirmed the good performance of both models (Tables 2 and 3). Other studies could show comparable sensitivity values of $91 \%$ for detecting gastric tumors [48] and an accuracy of $88 \%$ in a colorectal ex vivo study [49]. By using a PCA-DA approach, a good tissue group formation could be achieved. The high degree of similarities between our models demonstrates the great robustness of DF ELSS imaging.

\section{Validation of PCA-DA models with HE-staining}

A test set of ELS spectra was predicted by both PCA-DA models and identified either as EP, GM, or AT. The prediction outcome is compared with the HE diagnosis of identical tissue regions and verified whether the results match or not. For the Whiskbroom model, twelve tissue areas are expected to be AT. Eight of these regions were assigned to the AT cluster whereas the remaining four were diagnosed as EP tissue (Table 4). One explanation for this discrepancy in prediction is the tumor heterogeneity. Tumorous tissue cannot only consist of different tumor subpopulations, but also of histologically healthy areas $[35,50,51]$. Another plausible explanation could be that HNSCC tumors can exhibit variable levels of cellular differentiation. The same tumor can have regions of lower differentiation, possibly recognized as AT, whereas other, not healthy, regions are better differentiated and therefore classified as EP. A partial identification of the prediction regions as EP is thus possible. The corresponding HE diagnosis illustrates an overall AT impression and thus mostly coincide with the Whiskbroom results. Comparable outcomes were achieved by the Pushbroom model, which was able to assign 20 prediction regions out of 22 as AT tissue (Table 4). Only two areas were classified as EP and GM. The HE investigation confirmed that the tissue is modified. The two wrongly identified Pushbroom regions are once more explainable by healthy regions within an otherwise tumorous tissue [35, 52]. Additionally, two sets of EP spectra were tested against the Whiskbroom and Pushbroom models. The Whiskbroom PCA-DA was 
verified with four potential EP areas adjacent to an HEidentified carcinoma region (Table 4). These EP areas were chosen to investigate whether the tissue has already morphologically changed or is still intact. As a result, the model classified three of four EP regions as AT and only one was identified as epithelial tissue (Table 4). By close examination of the associated $\mathrm{HE}$ areas, all regions were categorized as AT. This comparison reveals how sensitive the ELS-based Whiskbroom model is in terms of detecting small morphological changes. Various EP regions were also assigned by the Pushbroom model. Four of six EP areas were attributed to the EP group and two of them were identified as GM (Table 4). The HE examination, however, revealed a distinct classification of all six test regions as EP. Both failing model predictions can be explained by the close vicinity of the GM tissue to the EP. For the GM prediction, two different testing regions were defined for the Whiskbroom and seven GM areas were chosen for the Pushbroom model (Table 4). Since the GM classification only plays a minor role, fewer ELS spectra were tested. Both GM regions were correctly allocated by the Whiskbroom PCA-DA, which also matches with the HE-evaluation (Table 4). Comparable results were achieved with the Pushbroom model and were additionally approved by the HE analysis. Here, seven out of seven test regions were identified as GM (Table 4). A high conformity with HE diagnosis was reached and thus the Whiskbroom and Pushbroom model predictions were proven reliable. The comparison of a statistical-based prediction model with the histopathology as the gold standard is a common approach and was also accomplished by others [53].

\section{Comparison of Whiskbroom and Pushbroom PCA-DA models}

Both imaging models demonstrated good predictive skills, which are in high accordance with the HE diagnosis. The model structures of the Whiskbroom and Pushbroom PCADA models are almost identical in terms of number and types of PCs. Besides, a great similarity was also depicted for all three loadings plots of both models (Fig. 5c, Fig. 6c). Although the acquisition principles and resolutions differ between Whiskbroom and Pushbroom imaging, comparable statistical models could be formed with almost identical values for accuracy, sensitivity, specificity, and precision (Table 2, Table 3). Based on the prediction results, the Pushbroom model seemingly achieved an overall better classification than the Whiskbroom model for all investigated tissue types (Table 4). In this context, some aspects need to be considered. First, the overall number of ELS test spectra was higher for the Pushbroom prediction in comparison to the Whiskbroom one. Therefore, one or two misclassifications by the Whiskbroom model have a significant higher impact on the relative prediction outcome than for the Pushbroom model with a greater testing population. This creates the impression of a better Pushbroom prediction prognosis compared to the Whiskbroom one and favors this model in terms of its prediction capability. Considering identical testing populations, the correct classification ability might change between both models. For this reason, a comparison of the model's prediction is difficult. Fortunately, a great amount of correctly predicted spectra and a small number of false classifications for both models occurred. Nevertheless, the differences of false prediction results between the models are minor and thus a clear capability preference for one or the other model is challenging to define. Second, the test regions of the EP margin turned out to be mostly AT as was confirmed by the Whiskbroom model and a detailed HE-diagnosis (Table 4). In further experiments, explicit EP regions need to be identified and examined by Whiskbroom imaging in order to validate whether the EP identification is fully possible. Still, the Whiskbroom results are reliable. The Pushbroom model testing, however, is thus more representative. Nevertheless, an enlarged testing of unknown tissue areas would be necessary to verify and confirm that the Pushbroom PCA-DA is the better model for the HNSCC application. An extension of the ELS training set for both models should also be realized to further advance their statistical validity. Additional improvements will encompass an expansion of the mouse model with a much higher amount of different $\mathrm{HN}$ tissue samples. Therefore, tissue heterogeneity will become less prominent from a statistical point of view.

For assessing whether the Whiskbroom or Pushbroom method is more suitable for the tissue classification, the different spatial and optical resolutions are considered. The applied spatial resolution for Whiskbroom imaging is equivalent to $7.5 \mu \mathrm{m}$. Using this resolution in combination with a confocal setup, no spatial information is mixed and an overview image of the investigated tissue extract is generated. Although higher spatial resolutions would have been possible with the instrumental setup, the detected spatial information content was sufficient to create an accurate Whiskbroom PCA-DA model. Based on this model, a high number of correct tissue assignments was achievable (Table 4). However, a high spatial resolution would be necessary to detect the small tissue-related differences in a single-point measurement fashion. This is particularly important for the recognition of tissue margins. Future experiments need to reveal whether the chosen spatial resolution is suitable for a potential tissue-border identification or if an adaption in resolution is required. For this study, most tissue classifications were performed with pathologically distinct tissue regions. Within this context, we thus demonstrated that the used Whiskbroom model is applicable to clearly classify these tissue regions, and our scanned images generated the spectral information necessary for this purpose. In order to resolve the spectral scattering 
patterns of each image, the high spectral resolution of $1.6 \mathrm{~nm}$ for the Whiskbroom setup is essential. The combination of this spectral resolution and the applied data preprocessing enabled the visualization and extraction of the Mie patterns with superimposed small ripples, as visualized by the loadings plots (Fig. 5c). Within the context of data preprocessing, a relatively high degree of spectral smoothing and a spatial averaging were applied. Both measures eliminated noise signals, but simultaneously maintained the superimposed small ripple patterns (Fig. 5c). Differences in tissue-section thicknesses were removed by a data normalization and are thus not represented by the scattering patterns (Fig. $5 \mathrm{c}$ ). In addition to the spatial and spectral resolution of the Whiskbroom imaging, the chosen spectral range of 412-975 nm might also play an important role in tissue classification. In this spectral region, the $3^{\text {rd }}$ overtone of the NIR region is present and influences not only the model's tissue cluster formation (Fig. 5b), but also the classification outcome of the tissue predictions. We ascertain the NIR region to be substantial for the PCA tissue cluster separation and also for the good classification results by the Whiskbroom model. Certainly, this also results from the high photon penetration in this spectral range. Compared to Whiskbroom imaging, the Pushbroom imager reveals a similar spectral resolution of $1.2 \mathrm{~nm}$, but higher spatial resolutions in $\mathrm{x}$ - and $\mathrm{y}$-direction. In $\mathrm{x}$-direction, the resolution is diffraction-limited whereas in y-direction it is equivalent to $1.5 \mu \mathrm{m}$. The resolution in $\mathrm{x}$-direction depends on the number of pixels along the axis, the microscope magnification power $(20 \times)$, and the resolution limit of the optics. In y-direction, the spatial resolution is additionally influenced by the experimentally defined step size of $0.3 \mu \mathrm{m}$. The combination of spatial resolution and step size in y-direction results in a scanning overlap of $1.2 \mu \mathrm{m}$ and thus five scanning steps cover identical spectral information. From a spatial perspective, Pushbroom images thus contain a much higher information content in comparison to Whiskbroom images. Each tissue section within the Pushbroom scan range is measured and small tissue differences can be gathered. Using this data, a robust Pushbroom PCA-DA model was formed and a high level of correct tissue predictions was accomplished (Table 4). We assume the high spatial resolutions to be much more appropriate for tissue-margin identification than the current Whiskbroom spatial resolution. In prospective experiments, the effect of the Pushbroom's spatial resolutions in terms of border recognition also needs to be studied. So far, the applied classifications only included border-free tissue regions which were successfully predicted by the Pushbroom model. Therefore, the overrepresentation of identical spectral information due to the scanning overlap does not seem to negatively affect the classification outcome, as most prediction areas were correctly assigned by the model. Nevertheless, the spatial resolution in y-direction as well as the step size needs to be adjusted in a way that all the spatial information is covered and no scan time is wasted on identical spots. Additionally, the impact on the scan direction of the tissue (east to west, north to south, and vice versa) needs to be investigated. In combination with the overrepresentation of identical spectra, the earlier described data preprocessing (spectral smoothing and spatial averaging) might have caused in this case a loss of spectral information. Due to the additional spatial averaging, smaller Mie patterns might have been eliminated and the overall shape is much smoother in comparison to the Whiskbroom patterns, illustrated by the loadings (Fig. 6c). Still, the obtained spectral Mie patterns are representative enough to allow a distinct PCA clustering and a high level of correctly assigned tissue classifications (Fig. 6b, Fig. 6c, Table 4). One positive effect of the spectral overrepresentation might be an increasing robustness of the Pushbroom PCA-DA model which might also be reflected by the classification results. Compared to the Whiskbroom imaging, the spectral range of the Pushbroom imaging encompassed 398-715 nm and thus the $3^{\text {rd }}$ overtone of the NIR region is not considered. This is a drawback of our specific Pushbroom imager since the NIR region has an important impact on the tissue cluster formation and classification results, as stated earlier for the Whiskbroom model. We assume that the large statistics of the Pushbroom model compensates for the missing spectral impact of the NIR region and thus generates an even better model performance and classification ability compared to the Whiskbroom model.

Considering all the previously mentioned arguments, we assess the Pushbroom imaging to be more suitable for the detection and classification of our mouse tissue samples in comparison to Whiskbroom imaging. The authors point out that Pushbroom imaging can only be better suitable for a specific application than Whiskbroom imaging if the scanning overlap and spectral overrepresentation is optimized with an appropriate setup. Consequently, Pushbroom imaging as a fast, easy-applicable, and low-cost instrument might be applicable as a prospective diagnostic tool in a clinical daily routine.

\section{Conclusion}

In conclusion, we successfully adapted two spectroscopic setups with a DF modulation to improve the detection of ELS and enabled the application of DF ELSS as imaging modality. One of the imaging systems was based on a Whiskbroom point-by-point scanning whereas the other was equipped with a Pushbroom imager to record ELS spectra in two different manners. Both imaging methods were employed on a HNSCC mouse model in a proof-of-concept study and generated a valuable ELS spectra set of different lingual tissue types. The ELS data were treated with several pre-processing steps to eliminate confounding factors of the sample or during the measurements. By assessing the treated ELS data with a PCA and a subsequent DA, we investigated whether DF ELSS imaging is capable of discriminating different tissue types and if either Whiskbroom or Pushbroom detection might be more suitable for 
this purpose. Our results show that DF ELSS is a very sensitive imaging technique able to distinguish between EP, GM, and AT based on their characteristic ELS pattern. PCAs for Whiskbroom and Pushbroom imaging revealed a distinct separation and thus differentiation of the three tissue type groups, although the Pushbroom PCA achieved a better cluster segregation. ELS is directly linked to the tissue's morphology and thus the PCA separation is based on morphological changes between the tissues. Using both detection principles, accurate PCA-DA models were generated with which model-unknown ELS spectra were predicted and matched with the corresponding HE-staining. These results demonstrated that Whiskbroom and Pushbroom predictions are largely consistent with the histopathological evaluation. Nevertheless, we define the Pushbroom method to be more suitable for these samples with their specific absorption and scattering properties investigated in this study. Although the main goal of our study was the comparison of two DF ELSS imaging techniques and their suitability in HNSCC differentiation, the possible application of a Pushbroom setup as a non-destructive, cheap, and high-throughput technique in a clinical daily routine should be emphasized.

Supplementary Information The online version contains supplementary material available at https://doi.org/10.1007/s00216-021-03726-5.

\begin{abstract}
Acknowledgements We thank Fiona Watt and her lab (King's College London) as well as the King's College London Biological Services Unit for support with the mouse experiments. Additionally, our thanks go to Garrit Koller (King's College London Dental Institute) for helpful discussions and his support for our collaboration. Furthermore, we thank Barbara Boldrini (Reutlingen University) for her supporting contributions as an expert in hyperspectral imaging as well as Ashutosh Mukherjee (Reutlingen University) for his large expertise in the WITec Alpha 300 RS confocal system and all his helpful discussions concerning the instrumental setups. Last but not least, we thank Tim Rammler (University of Tübingen) for adjusting the Pushbroom microscope system and are also grateful for all the helpful discussions and his expertise in this field.
\end{abstract}

Funding Open Access funding enabled and organized by Projekt DEAL. Inês Sequeira received the support of a Barts Charity Lectureship (grant MGU045). Additionally, this study was funded by the doctoral program of the University of Tübingen and Reutlingen University "Intelligente Prozess- und Materialentwicklung in der Biomateriomics (IPMB)" which is supported by the MWK Baden-Württemberg ( $\mathrm{PhD}$ student fellowship to MCB and MS).

Data availability The data sets generated and/or analyzed during the current study are not publicly available since they are part of ongoing $\mathrm{PhD}$ theses. However, the data sets can be accessible on reasonable request from the corresponding author.

\section{Declarations}

Ethics approval All animal procedures were subject to institutional ethical review and approved by the UK Home Office (in accordance with UK law, Animals Scientific Procedures Act 1986) at King's College London prior to commencement (Project license number 70/8474). We adhere to ARRIVE guidelines as set out by the NC3Rs.
Conflict of interest The authors declare no competing interests.

Open Access This article is licensed under a Creative Commons Attribution 4.0 International License, which permits use, sharing, adaptation, distribution and reproduction in any medium or format, as long as you give appropriate credit to the original author(s) and the source, provide a link to the Creative Commons licence, and indicate if changes were made. The images or other third party material in this article are included in the article's Creative Commons licence, unless indicated otherwise in a credit line to the material. If material is not included in the article's Creative Commons licence and your intended use is not permitted by statutory regulation or exceeds the permitted use, you will need to obtain permission directly from the copyright holder. To view a copy of this licence, visit http://creativecommons.org/licenses/by/4.0/.

\section{References}

1. Krafft C, Schmitt M, Schie IW, Cialla-May D, Matthäus C, Bocklitz T, Popp J. Label-free molecular imaging of biological cells and tissues by linear and nonlinear Raman spectroscopic approaches. Angew Chem Int Ed. 2017;56(16):4392-430. https:// doi.org/10.1002/anie.201607604.

2. Mian SA, Yorucu C, Ullah MS, Rehman IU, Colley HE. Raman spectroscopy can discriminate between normal, dysplastic and cancerous oral mucosa: a tissue-engineering approach. J Tissue Eng Regen Med. 2017;11(11):3253-62. https://doi.org/10.1002/ term.2234.

3. Kumar S, Srinivasan A, Nikolajeff F. Role of infrared spectroscopy and imaging in cancer diagnosis. Curr Med Chem. 2018;25(9):1055-72.

4. Bray F, Ferlay J, Soerjomataram I, Siegel RL, Torre LA, Jemal A. Global cancer statistics 2018: GLOBOCAN estimates of incidence and mortality worldwide for 36 cancers in 185 countries. CA Cancer J Clin. 2018;68(6):394-424. https://doi.org/ 10.3322/caac.21492.

5. Alsahafi E, Begg K, Amelio I, Raulf N, Lucarelli P, Sauter T, Tavassoli M. Clinical update on head and neck cancer: molecular biology and ongoing challenges. Cell Death Dis. 2019;10(8):540. https://doi.org/10.1038/s41419-019-1769-9.

6. Ferlay J, Colombet M, Soerjomataram I, Mathers C, Parkin DM, Piñeros M, Znaor A, Bray F. Estimating the global cancer incidence and mortality in 2018: GLOBOCAN sources and methods. Int J Cancer. 2018;144(8):1941-53. https://doi.org/10. 1002/ijc. 31937.

7. Sano D, Myers JN. Metastasis of squamous cell carcinoma of the oral tongue. Cancer Metastasis Rev. 2007;26(3):645-62. https://doi.org/10.1007/s10555-007-9082-y.

8. Sequeira I, Rashid M, Tomás IM, Williams MJ, Graham TA, Adams DJ, Vigilante A, Watt FM. Genomic landscape and clonal architecture of mouse oral squamous cell carcinomas dictate tumour ecology. Nat Commun. 2020;11(1):5671. https:// doi.org/10.1038/s41467-020-19401-9.

9. Tan NCW, Herd MK, Brennan PA, Puxeddu R. The role of narrow band imaging in early detection of head and neck cancer. Br J Oral Maxillofac Surg. 2012;50(2):132-6. https://doi.org/ 10.1016/j.bjoms.2010.12.001.

10. Devpura S, Thakur JS, Sethi S, Naik VM, Naik R. Diagnosis of head and neck squamous cell carcinoma using Raman spectroscopy: tongue tissues. J Raman Spectrosc. 2012;43(4):490-6. https://doi.org/10.1002/jrs.3070.

11. Dhingra JK, Zhang X, McMillan K, Kabani S, Manoharan R, Itzkan I, Feld MS, Shapshay SM. Diagnosis of head and neck precancerous lesions in an animal model using fluorescence 
spectroscopy. Laryngoscope. 1998;108(4):471-5. https://doi. org/10.1097/00005537-199804000-00002.

12. Roy HK, Liu Y, Wali RK, Kim YL, Kromine AK, Goldberg MJ, Backman V. Four-dimensional elastic light-scattering fingerprints as preneoplastic markers in the rat model of colon carcinogenesis. Gastroenterology. 2004;126(4):1071-81. https:// doi.org/10.1053/j.gastro.2004.01.009.

13. $\mathrm{Xu} \mathrm{M,} \mathrm{Wu} \mathrm{T,} \mathrm{Qu} \mathrm{J.} \mathrm{Unified} \mathrm{Mie} \mathrm{and} \mathrm{fractal} \mathrm{scattering} \mathrm{by} \mathrm{cells}$ and experimental study on application in optical characterization of cellular and subcellular structures. J Biomed Opt. 2008;13(2):024015.

14. Kim YL, Yang L, Wali RK, Roy HK, Goldberg MJ, Kromin AK, Kun C, Backman V. Simultaneous measurement of angular and spectral properties of light scattering for characterization of tissue microarchitecture and its alteration in early precancer. IEEE J Sel Top Quantum Electron. 2003;9(2):243-56. https:// doi.org/10.1109/JSTQE.2003.814183.

15. Bigio I, Bown S, Briggs G, Kelley C, Lakhani S, Pickard D, Ripley P, Rose I, Saunders C. Diagnosis of breast cancer using elastic-scattering spectroscopy: preliminary clinical results. Journal of Biomedical Optics. 2000;5(2):221-8. https://doi. org/10.1117/1.429990

16. Canpolat M, Gökhan AG, Çiftçioğlu MA, Erin N. Differentiation of melanoma from non-cancerous tissue in an animal model using elastic light single-scattering spectroscopy. Technol Cancer Res Treat. 2008;7(3):235-40. https://doi.org/10.1177/15330 3460800700309.

17. Gong J, Yi J, Turzhitsky VM, Muro K, Li X. Characterization of malignant brain tumor using elastic light scattering spectroscopy. Dis Markers. 2008;25(6):303-12. https://doi.org/10.1155/ 2008/208120.

18. Müller MG, Valdez TA, Georgakoudi I, Backman V, Fuentes C, Kabani S, Laver N, Wang Z, Boone CW, Dasari RR, Shapshay SM, Feld MS. Spectroscopic detection and evaluation of morphologic and biochemical changes in early human oral carcinoma. Cancer. 2003;97(7):1681-92. https://doi.org/10.1002/ cncr.11255.

19. Stefanakis M, Lorenz A, Bartsch JW, Bassler MC, Wagner A, Brecht M, Pagenstecher A, Schittenhelm J, Boldrini B, Hakelberg S, Noell S, Nimsky C, Tatagiba M, Ritz R, Rebner K, Ostertag E. Formalin fixation as tissue preprocessing for multimodal optical spectroscopy using the example of human brain tumour cross sections. J Spectrosc. 2021;2021:5598309. https://doi.org/10.1155/ 2021/5598309.

20. Boldrini B, Kessler W, Rebner K, Kessler RW. Hyperspectral imaging: a review of best practice, performance and pitfalls for in-line and on-line applications. J Near Infrared Spectrosc. 2012;20(5):483-508.

21. Halicek M, Fabelo H, Ortega S, Callico GM, Fei B. In-vivo and ex-vivo tissue analysis through hyperspectral imaging techniques: revealing the invisible features of cancer. Cancers. 2019;11(6):756.

22. Basantia N, Nollet LM, Kamruzzaman M (2018) Hyperspectral Imaging Analysis and Applications for Food Quality. 1st edn. CRC Press, Boca Raton. https://doi.org/10.1201/9781315209203

23. Halicek M, Little JV, Wang X, Patel M, Griffith CC, El-Deiry MW, Chen AY, Fei B. Optical biopsy of head and neck cancer using hyperspectral imaging and convolutional neural networks. Proc SPIE Int Soc Opt Eng. 2019;10469:104690X. https://doi.org/ 10.1117/12.2289023.

24. Chung H, Lu G, Tian Z, Wang D, Chen ZG, Fei B Superpixelbased spectral classification for the detection of head and neck cancer with hyperspectral imaging. In: Medical Imaging 2016: Biomedical Applications in Molecular, Structural, and Functional Imaging, San Diego. International Society for Optics and Photonics. 2016. p 978813. https://doi.org/10.1117/12.2216559.
25. Baker MJ, Trevisan J, Bassan P, Bhargava R, Butler HJ, Dorling KM, Fielden PR, Fogarty SW, Fullwood NJ, Heys KA, Hughes C, Lasch P, Martin-Hirsch PL, Obinaju B, Sockalingum GD, SuléSuso J, Strong RJ, Walsh MJ, Wood BR, Gardner P, Martin FL. Using Fourier transform IR spectroscopy to analyze biological materials. Nat Protoc. 2014;9(8):1771-91. https://doi.org/10. 1038/nprot.2014.110.

26. Ostertag E, Stefanakis M, Rebner K, Kessler RW. Elastic and inelastic light scattering spectroscopy and its possible use for labelfree brain tumor typing. Anal Bioanal Chem. 2017;409(28):661323. https://doi.org/10.1007/s00216-017-0614-1.

27. Rebner K, Schmitz M, Boldrini B, Kienle A, Oelkrug D, Kessler RW. Dark-field scattering microscopy for spectral characterization of polystyrene aggregates. Opt Express. 2010;18(3):3116-27. https://doi.org/10.1364/OE.18.003116.

28. Li Q, He X, Wang Y, Liu H, Xu D, Guo F. Review of spectral imaging technology in biomedical engineering: achievements and challenges. J Biomed Opt. 2013;18(10):100901.

29. Wallenius K, Lekholm U. Oral cancer in rats induced by the water-soluble carcinogen 4-nitrochinoline $\mathrm{N}$-oxide. Odontol Revy. 1973;24(1):39-48.

30. Sequeira I, Neves JF, Carrero D, Peng Q, Palasz N, Liakath-Ali K, Lord GM, Morgan PR, Lombardi G, Watt FM. Immunomodulatory role of Keratin 76 in oral and gastric cancer. Nat Commun. 2018;9(1):3437. https://doi.org/10.1038/s41467-018-05872-4.

31 Downes DJ, Chonofsky M, Tan K, Pfannenstiel BT, Reck-Peterson SL, Todd RB. Characterization of the mutagenic spectrum of 4-nitroquinoline 1-oxide (4-NQO) in Aspergillus nidulans by whole genome sequencing. G3 (Bethesda). 2014;4(12):2483-92. https://doi.org/10.1534/g3.114.014712.

32. Vered M, Yarom N, Dayan D. 4NQO oral carcinogenesis: animal models, molecular markers and future expectations. Oral Oncol. 2005;41(4):337-9. https://doi.org/10.1016/j.oraloncology.2004. 07.005 .

33. Tang X-H, Knudsen B, Bemis D, Tickoo S, Gudas LJ. Oral cavity and esophageal carcinogenesis modeled in carcinogen-treated mice. Clin Cancer Res. 2004;10(1):301-13. https://doi.org/10. 1158/1078-0432.Ccr-0999-3.

34 Slootweg PJ, El-Naggar AK. World Health Organization 4th edition of head and neck tumor classification: insight into the consequential modifications. Virchows Arch. 2018;472(3):311-3. https://doi.org/10.1007/s00428-018-2320-6.

35. Fidler I, Hart I. Biological diversity in metastatic neoplasms: origins and implications. Science. 1982;217(4564):998-1003. https://doi.org/10.1126/science.7112116.

36. Lu G, Little JV, Wang X, Zhang H, Patel MR, Griffith CC, ElDeiry MW, Chen AY, Fei B. Detection of head and neck cancer in surgical specimens using quantitative hyperspectral imaging. Clin Cancer Res. 2017;23(18):5426-36. https://doi.org/10.1158/ 1078-0432.Ccr-17-0906.

37. El-Naggar AK, Chan JKC, Grandis JR, Takata T, Slootweg PJ (2017) WHO Classification of Head and Neck Tumours, vol 9th. IARC WHO Classification of Tumours, 4th edn. International Agency for Research on Cancer, Lyon.

38. Perelman LT, Backman V, Wallace M, Zonios G, Manoharan R, Nusrat A, Shields S, Seiler M, Lima C, Hamano T, Itzkan I, Van Dam J, Crawford JM, Feld MS. Observation of periodic fine structure in reflectance from biological tissue: a new technique for measuring nuclear size distribution. Phys Rev Lett. 1998;80(3):627-30. https://doi.org/10.1103/PhysRevLett.80. 627.

39. Choi J, Choo J, Chung H, Gweon D-G, Park J, Kim HJ, Park S, Oh $\mathrm{C}-\mathrm{H}$. Direct observation of spectral differences between normal and basal cell carcinoma (BCC) tissues using confocal Raman microscopy. Biopolymers. 2005;77(5):264-72. https://doi.org/10. 1002/bip.20236. 
40. Favreau PF, Deal JA, Harris B, Weber DS, Rich TC, Leavesley SJ. Label-free spectroscopic tissue characterization using fluorescence excitation-scanning spectral imaging. J Biophotonics. 2020;13(2):e201900183. https://doi.org/10.1002/jbio.201900183.

41 Jolliffe I. Principal component analysis Springer Series in Statistics. 2nd ed. New York: Springer-Verlag, New York; 2002. https:// doi.org/10.1007/b98835.

42. Wold S, Esbensen K, Geladi P. Principal component analysis. Chemom Intell Lab Syst. 1987;2(1):37-52. https://doi.org/10. 1016/0169-7439(87)80084-9.

43. Chen Y, Zhu S, Fu S, Li Z, Huang F, Yin H, Chen Z. Classification of hyperspectral images for detection of hepatic carcinoma cells based on spectral-spatial features of nucleus. J Innov Opt Health Sci. 2020;13(01):2050002. https://doi.org/10.1142/s179354582 0500029.

44. Backman V, Wallace MB, Perelman LT, Arendt JT, Gurjar R, Müller MG, Zhang Q, Zonios G, Kline E, McGillican T, Shapshay S, Valdez T, Badizadegan K, Crawford JM, Fitzmaurice M, Kabani S, Levin HS, Seiler M, Dasari RR, Itzkan I, Van Dam J, Feld MS. Detection of preinvasive cancer cells. Nature. 2000;406(6791):356. https://doi.org/10.1038/35017638.

45. Jain PK, Lee KS, El-Sayed IH, El-Sayed MA. Calculated absorption and scattering properties of gold nanoparticles of different size, shape, and composition: applications in biological imaging and biomedicine. J Phys Chem B. 2006;110(14):7238-48. https:// doi.org/10.1021/jp057170o.

46. Schmitt JM, Kumar G. Optical scattering properties of soft tissue: a discrete particle model. Appl Opt. 1998;37(13):2788-97. https:// doi.org/10.1364/AO.37.002788.

47. Kumar G, Schmitt JM Micro-optical properties of tissue. In: Advances in Laser and Light Spectroscopy to Diagnose Cancer and Other Diseases III: Optical Biopsy, San Jose. International Society for Optics and Photonics. 2016. p 106-16. https://doi.org/ $10.1117 / 12.237578$.

48. Akbari H, Uto K, Kosugi Y, Kojima K, Tanaka N. Cancer detection using infrared hyperspectral imaging. Cancer Sci. 2011;102(4):852-7. https://doi.org/10.1111/j.1349-7006.2011. 01849.x.

49. Baltussen EJ, Kok EN, Brouwer de Koning S, Sanders J, Aalbers AG, Kok NF, Beets G, Flohil C, Bruin S, Kuhlmann KF, Sterenborg HJC, Ruers TJ. Hyperspectral imaging for tissue classification, a way toward smart laparoscopic colorectal surgery. J Biomed Opt. 2019;24(1):016002.

50 Marusyk A, Polyak K. Tumor heterogeneity: causes and consequences. Biochim Biophys Acta Rev Cancer. 2010;1805(1)10517.https://doi.org/10.1016/j.bbcan.2009.11.002.

51. Jacob R, Welkoborsky HJ, Mann WJ, Höfken F, Dienes HP, Freije JE. Heterogeneity of squamous cell carcinomas of the head and neck-analysis of tumor biologic factors and proliferation rates. Laryngoscope. 1996;106(9):1170-5. https://doi.org/10.1097/ 00005537-199609000-00023.

52. Egeblad M, Nakasone ES, Werb Z. Tumors as organs: complex tissues that interface with the entire organism. Dev Cell. 2010;18(6):884-901. https://doi.org/10.1016/j.devcel.2010.05. 012 .

53. Fei B, Lu G, Wang X, Zhang H, Little JV, Patel MR, Griffith CC, El-Diery MW, Chen AY. Label-free reflectance hyperspectral imaging for tumor margin assessment: a pilot study on surgical specimens of cancer patients. J Biomed Opt. 2017;22(8):1-7. https://doi.org/10.1117/1.JBO.22.8.086009.

Publisher's note Springer Nature remains neutral with regard to jurisdictional claims in published maps and institutional affiliations.

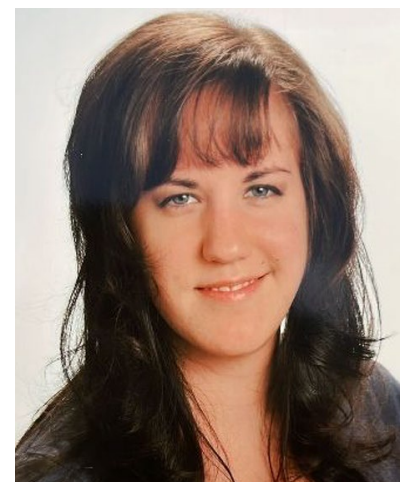

Miriam C. Bassler is a PhD student in physical and theoretical chemistry at the University of Tübingen. She works at the research center Process Analysis and Technology (PA\&T) at Reutlingen University. Her research focuses on the bioanalytical analysis of single cells, tumor spheroids, and tissues by spectroscopic imaging and multivariate data analysis.

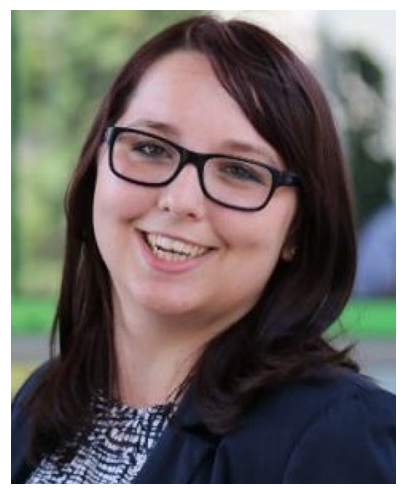

Mona Stefanakis is a PhD student in physical and theoretical chemistry at the University of Tübingen. She works at the research center Process Analysis and Technology (PA\&T) at Reutlingen University. Her research interests include hyperspectral imaging and multivariate data analysis.

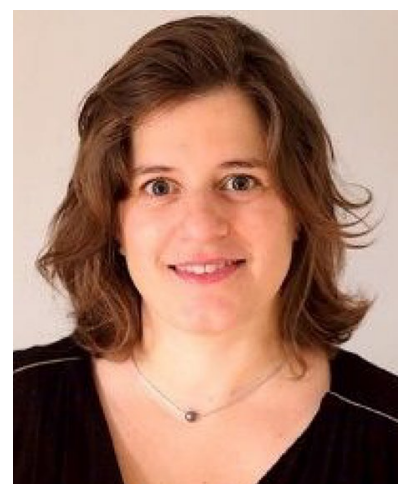

Inês Sequeira is Group Leader and Assistant Professor at Queen Mary University London. As a stem cell biologist, she has dedicated much of her research career to studying skin and oral epithelial homeostasis. Her lab's research focuses on understanding the molecular mechanisms involved in oral mucosa scarless wound healing and on oral cancer. 


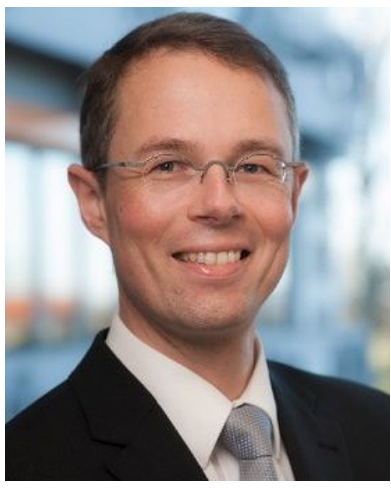

Edwin Ostertag is Senior Scientist at the research center Process Analysis and Technology (PA\&T) of Reutlingen University. He received his $\mathrm{PhD}$ in physical chemistry from the University of Tübingen. His current research focuses on optical spectroscopy for the characterization of biomaterials and the assessment of food quality. He is further interested in medical technology and blockchain use cases.

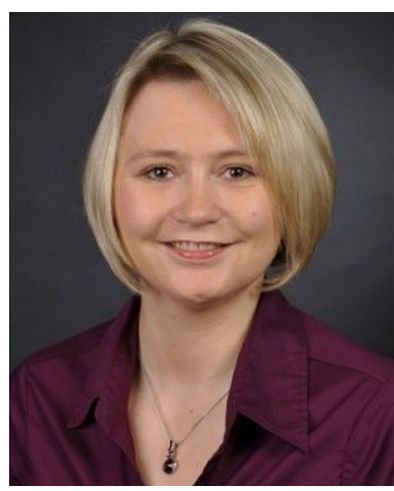

Alexandra Wagner is a $\mathrm{PhD}$ student in physical and theoretical chemistry at the University of Tübingen. She works at the research center Process Analysis and Technology (PA\&T) at Reutlingen University. Her research interests include optical spectroscopy, chemical imaging, and multivariate data analysis.

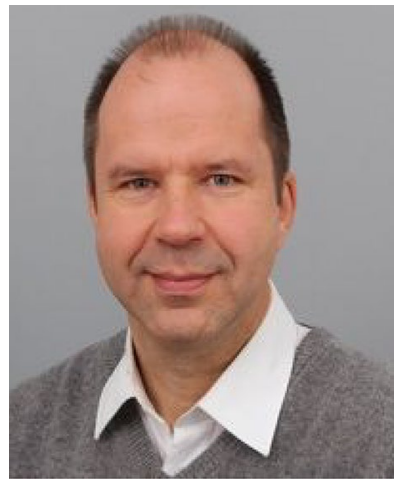

Jörg W. Bartsch is Head of the Neurosurgery laboratory at Philipps University Marburg and is currently Coordinator of the ERANET Project "PerProGlio." His research is focused on multiple aspects in translational oncology, ranging from spectroscopy to molecular gene analysis and drug discovery.

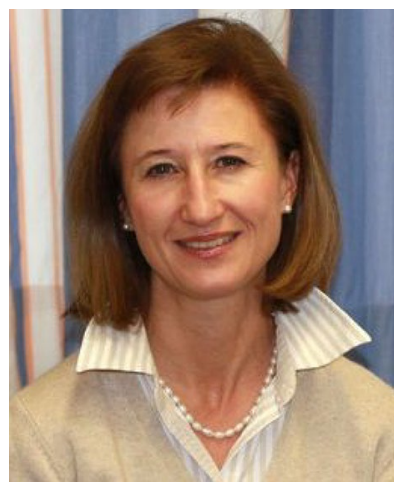

Marion Roeßler is Senior Pathologist at the Department of Pathology at the Philipps University of Marburg. Her current research includes the optimization and evaluation of digital images for head and neck squamous cell carcinomas (HNSCC) and cell cultures with a special focus on epithelial cell adhesion structures of normal and tumorous stroma. Further research is focused on breast cancer.

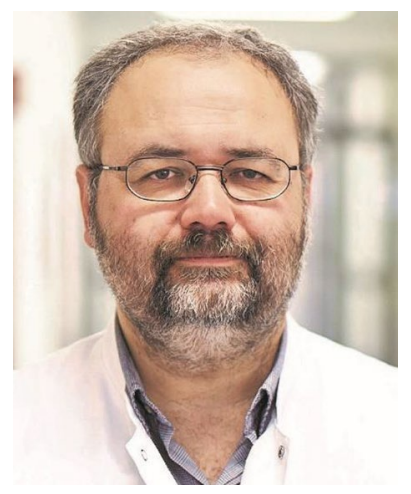

Robert Mandic is an attending physician and Director of the Interdisciplinary Head and Neck Oncology Laboratory at the Department of Otorhinolaryngology at the University Hospital in Marburg, Germany. His research is focusing on candidate genes and mechanisms involved in therapy resistance of head and neck squamous cell carcinomas (HNSCC) representing the most frequent malignancies of the upper aero-digestive tract.

Eike F. Reddmann is a former Bachelor student at the research center Process Analysis and Technology (PA\&T), Reutlingen University. His research interests include interdisciplinary approaches in cancer detection and therapy.

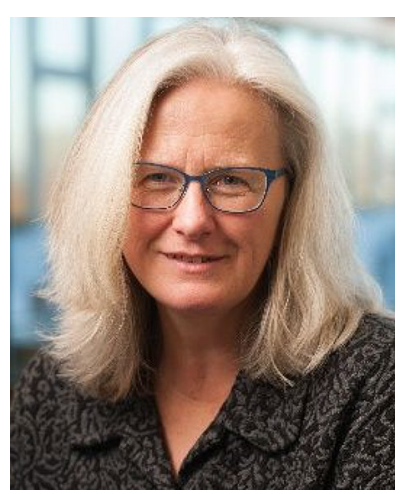

Anita Lorenz received her $\mathrm{PhD}$ in chemistry from the RWTH Aachen University. She is Senior Scientist at the research center Process Analysis and Technology (PA\&T) at Reutlingen University. Her current work focuses on optical spectroscopy, hyperspectral imaging techniques, nano- and microspectroscopy, 3D-microprinting, and multivariate data analysis.

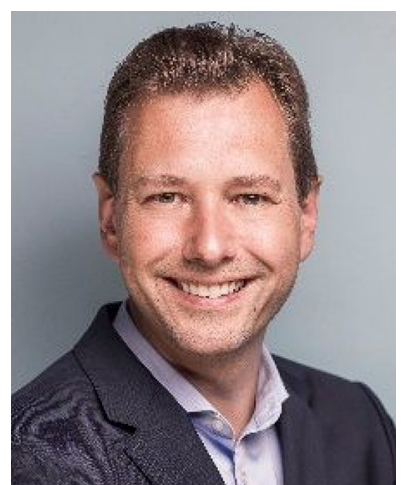

Karsten Rebner is Professor for Photonics and Process Analytical Technology in the Faculty of Applied Chemistry at Reutlingen University. He obtained his $\mathrm{PhD}$ degree from the University of Tübingen, Institute of Physical and Theoretical Chemistry. The focus of his current research is online optical spectroscopy and multivariate data analysis of biological and functional materials. 


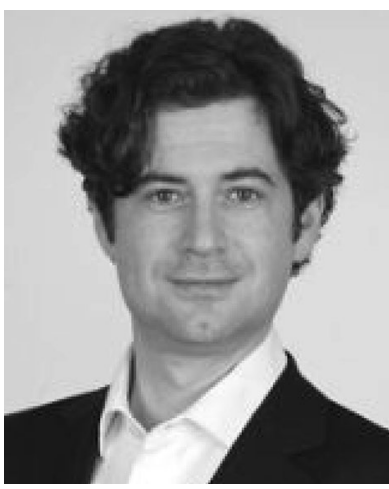

Marc Brecht studied physics in Tübingen and Berlin. Since 2016 he has been Full Professor of Experimental Physics at Reutlingen University. In addition, he serves as Bridging Professor between Reutlingen University and the University of Tübingen. The scientific main focus of his work is optics, here in particular high-resolution microspectroscopy. 